\title{
Structural Determinants of Post-translational Modification and Catalytic Specificity for the Lipoyl Domains of the Pyruvate Dehydrogenase Multienzyme Complex of Escherichia coli
}

\author{
D. Dafydd Jones, H. James Horne, Pedro A. Reche \\ and Richard N. Perham*
}

Cambridge Centre for

Molecular Recognition

Department of Biochemistry

University of Cambridge

80 Tennis Court Road

Cambridge CB2 1GA, UK

${ }^{*}$ Corresponding author
The lipoyl domains of the dihydrolipoyl acyltransferase (E2p, E2o) components of the pyruvate and 2-oxoglutarate dehydrogenase multienzyme complexes are specifically recognised by their cognate 2-oxo acid decarboxylase (E1p, E1o). A prominent surface loop links the first and second $\beta$ strands in all lipoyl domains, close in space to the lipoyl-lysine $\beta$-turn. This loop was subjected to various modifications by directed mutagenesis of a sub-gene encoding a lipoyl domain of Escherichia coli E2p. Deletion of the loop (four residues) rendered the domain incapable of reductive acetylation by E. coli E1p in the presence of pyruvate, but insertion of a new loop (six residues) corresponding to that in the E2o lipoyl domain partly restored this ability, albeit with a much lower rate. However, the modified domain remained unable to undergo reductive succinylation by E1o in the presence of 2-oxoglutarate. Additional exchange of the two residues on the C-terminal side of the loop (V14A, E15T) had no effect. Insertion of a different four-residue loop also restored a limited ability to undergo reductive acetylation, but still significantly less than that of the wild-type domain. Exchanging the residue on the N-terminal side of the lipoyl-lysine $\beta$-turn in the E2p and E2o domains (G39T), both singly and in conjunction with the loop exchange, had no effect on the ability of the E2p domain to be reductively acetylated but did confer a slight increase in susceptibility to reductive succinylation. All mutant E2p domains, apart from that with the loop deletion (LD), were readily lipoylated in vitro by E. coli lipoate protein ligase A; the E2p LD mutant could be lipoylated only at a significantly lower rate. Likewise, this domain exhibited 1D and 2D NMR spectra characteristic of a partially folded protein, whereas the spectra of mutants with modified loops were similar to those of the wild-type domain. The surface loop is evidently important to the structural integrity of the domain and may help to stabilize the thioester bond linking the acyl group to the reduced lipoyl-lysine swinging arm as part of the catalytic mechanism. Recognition of the lipoyl domain by its partner E1 appears to be a complex process and not attributable to any single determinant on the domain.

Keywords: lipoyl domain; protein-protein interaction; post-translational modification; pyruvate dehydrogenase; N-Met processing

Present addresses: D.D. Jones, MRC Centre for Protein Engineering, Hills Road, Cambridge CB2 2QH, UK; H.J. Horne, St Vincent's Institute of Medical Research, 41 Victoria Parade, Fitzroy 3065, Melbourne, Victoria, Australia; P.A. Reche, DNAX Research Institute, 901 California Ave, Palo Alto, CA 94304-1104, USA.

Abbreviations used: E1, 2-oxo acid decarboxylase; E2, dihydrolipoyl acyltransferase; o, 2-oxoglutarate; p, pyruvate; LS, loop swap; LS+, loop swap plus V15AE16T mutation; LD, loop deletion; LplA, lipoate protein ligase A; Met-AP, methionyl aminopeptidase; N-Met, N-terminal methionine; NOESY, nuclear Overhauser effect spectroscopy.

E-mail address of the corresponding author: r.n.perham@bioc.cam.ac.uk 


\section{Introduction}

The 2-oxo acid dehydrogenase multienzyme complexes catalyse the oxidative decarboxylation of 2-oxo acids, generating NADH and the corresponding acyl coenzyme A. The three main members of the family are the pyruvate (PDH), 2oxoglutarate (2OGDH) and branched-chain 2-oxo acid dehydrogenase complexes, the substrates for the latter being the 2-oxo acids produced by the transamination of valine, leucine and isoleucine. In the PDH complex, the three component enzymes are pyruvate decarboxylase (E1p; EC 1.2.4.1), dihydrolipoyl acetyltransferase (E2p; EC 2.3.1.12) and dihydrolipoyl dehydrogenase (E3; EC 1.8.1.4). Corresponding enzymes are found in the $2 \mathrm{OGDH}$ and branched chain 2-oxo acid dehydrogenase complexes (reviewed by Perham, 1991; Mattevi et al., 1992a; Berg \& de Kok, 1997). E1 catalyses the initial decarboxylation of the 2-oxo acid, using thiamin diphosphate (ThDP) as a cofactor, and then reductively acylates a lipoyl group bound in $N^{6}$-amide linkage to a lysine residue in E2. E2 is an acyltransferase responsible for transferring the acyl group on to CoA, and the dihydrolipoyl group left on E2 is finally reoxidized to the dithiolane ring by the flavoprotein E3, with $\mathrm{NAD}^{+}$as the ultimate electron acceptor. E1 and E2 differ from complex to complex, whereas E3 is generally common to the different complexes in a given organism.

The structural core is provided by E2, with E1 and E3 bound around the periphery. The E2 chains are highly segmented comprising, from the $\mathrm{N}$ terminus, one to three lipoyl domains (ca $9 \mathrm{kDa}$ ), a peripheral subunit-binding domain (ca $4 \mathrm{kDa}$ ), and an acyltransferase domain (ca $28 \mathrm{kDa}$ ). In the PDH complex from Escherichia coli and other Gram-negative bacteria, each E2p chain contains three lipoyl domains and the inner core is formed by an assembly of 24 acetyltransferase domains arranged with octahedral symmetry. The E2o chain of the 2OGDH complex is very similar, except that it contains only one lipoyl domain. In contrast, the E2p chain from the PDH complex of Bacillus stearothermophilus, a Gram-positive organism, also contains only one lipoyl domain but the acetyltransferase domains aggregate to form a 60-mer icosahedral core (reviewed by Reed \& Hackert (1990) and Perham (1991)). In all these cases, the domains in the E2 chains are separated by long (25-30 residue) segments of polypeptide chain characteristically rich in alanine, proline and charged amino acids, which form flexible but extended linker regions, as judged by NMR spectroscopy (Green et al., 1992; Turner et al., 1993; and references therein).

Three-dimensional structures of all these E2 domains have been solved in recent years, among them the octahedral cores of the A. vinelandii PDH (Mattevi et al., 1992b) and E. coli 2OGDH (Knapp et al., 1998) complexes and the icosahedral cores of the Enterococcus faecalis and B. stearothermophilus PDH (Izard et al., 1999) complexes, by means of Xray crystallography; the peripheral subunit-binding domains of the E2 chains of the E. coli 2OGDH (Robien et al., 1992) and B. stearothermophilus PDH (Kalia et al., 1992) complexes by NMR spectroscopy; and lipoyl domains from the E2 chains of the B. stearothermophilus (Dardel et al., 1993) and human (Howard et al., 1998) PDH complexes, E. coli PDH (Green et al., 1995a; D.D.J. et al., unpublished work) and 2OGDH (Ricaud et al., 1996) complexes, and A. vinelandii PDH (Berg et al., 1997) and 2OGDH (Berg et al., 1996) complexes, also by NMR spectroscopy. One of the key features of these enzymes that can now be tackled is that of the protein-protein interactions that mediate their mechanisms and assembly. The first step in that direction is the determination of the structure of the complex between the peripheral subunit-binding domain of E2 and the E3 component of the B. stearothermophilus PDH complex (Mande et al., 1996).

Another key interaction is that between the E1 component and the lipoyl domain of E2. Free lipoate can act as a substrate for E2 and E3 but not for E1, whereas the lipoyl domain is highly effective as a substrate for reductive acylation, with the value of $k_{\text {cat }} / K_{\mathrm{m}}$ raised by a factor of $10^{4}$ for the PDH complex of E. coli (Reed et al., 1958; Graham et al., 1989). Moreover, in the E. coli (Graham et al., 1989) and A. vinelandii (Berg et al., 1998) PDH and 2OGDH complexes, the E2p and E2o lipoyl domains only function well as substrates for their respective E1p and E1o components. Thus, the lipoyl domain is crucial to a mechanism for "activating" the pendant lipoyl group as a substrate and to the system of substrate channelling that ensures reductive acylation is confined to a lipoyl group covalently attached to the intended E2 component (Perham, 1991; Perham \& Reche, 1998). The structures of the lipoyl domains from E. coli E2p and E2o are depicted in Figure 1. Their overall folds are identical, comprising a flattened $\beta$-barrel formed by two four-stranded $\beta$-sheets with a 2 -fold axis of quasi-symmetry. The lipoyl-lysine residue is found at the tip of a protruding $\beta$-turn, which may turn out to be important for its presentation to the active site of E1.

The means by which the E1 component "activates" the pendant lipoyl group for reductive acylation and exerts its specificity in acylating only lipoic acid bound to its partner E2 domain remains to be determined. The binding of the lipoyl domain to E. coli E1p is weak ( $K_{\mathrm{s}}$ not less than $1 \mathrm{mM}$ despite a $K_{\mathrm{m}}$ of ca $20 \mu \mathrm{M}$ ) and transient (Graham \& Perham, 1990). On the basis of directed mutagenesis and NMR spectroscopy (Wallis et al., 1996), residues in the prominent surface loop that links the first and second $\beta$-strands and lies close in space to the lipoyl-lysine $\beta$-turn (Figure 1 ) have been implicated in the interaction with E1p in the B. stearothermophilus PDH complex. We describe here a set of experiments with the E. coli E2p and E2o lipoyl domains, involving deletion and 


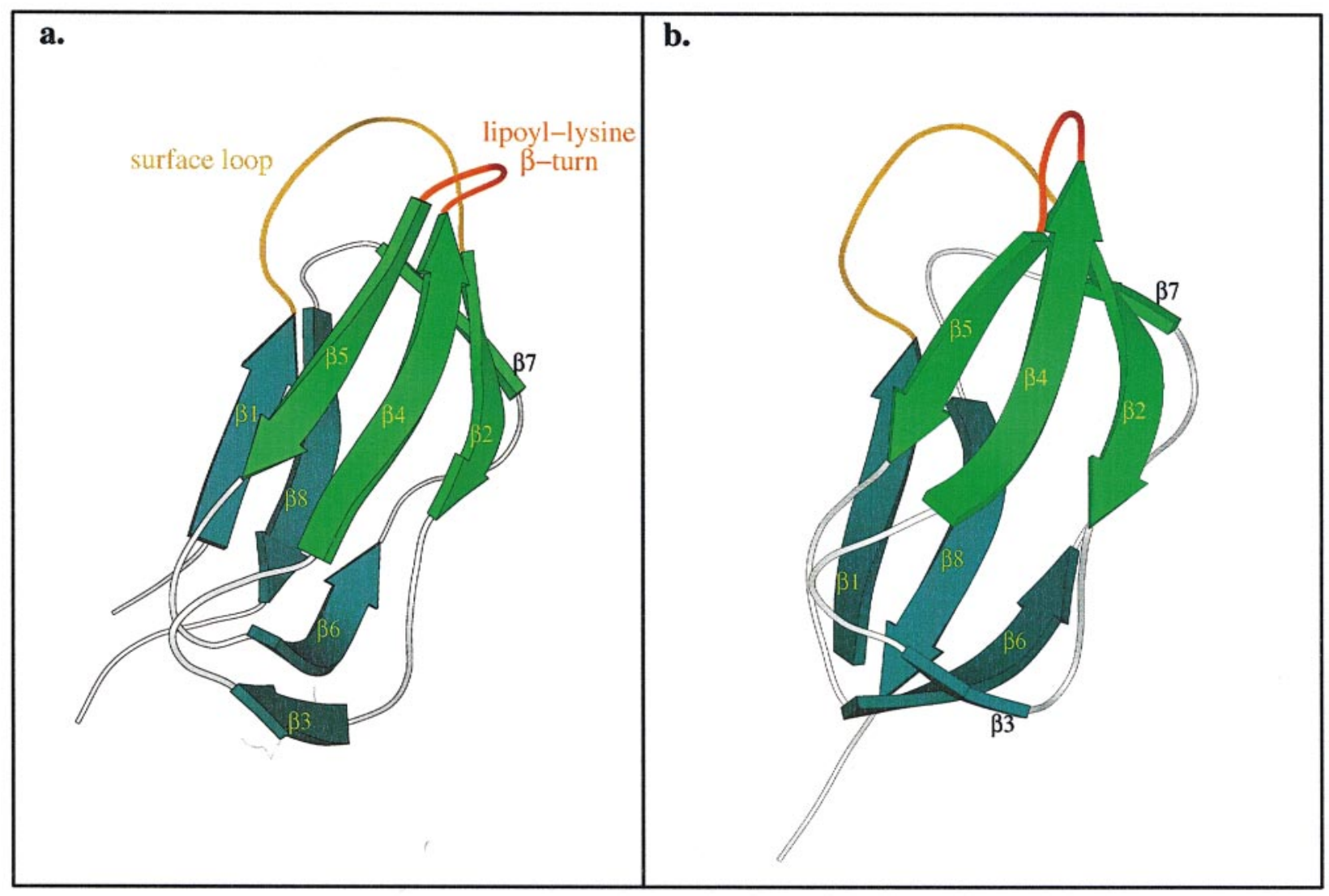

Figure 1. The three-dimensional structures of the E. coli (a) E2o (Ricaud et al., 1996) and (b) hybrid E2p (Green et al., 1995a) lipoyl domains. The lipoyl-lysine $\beta$-turn is indicated in red and the nearby surface loop linking the first and second $\beta$-strands is shown in orange. The two four-stranded $\beta$-sheets related by a 2 -fold axis of quasi-symmetry in both domains are shown in blue and green. The $\beta$-strands are numbered consecutively from the $\mathrm{N}$ terminus. The Figure was prepared using the program MOLSCRIPT (Kraulis, 1991).

mutation of residues in this surface loop, that indicate that the loop may be involved in the interaction with E1p and E1o but that it is not the sole determinant of specificity in reductive acylation. It appears that subtle differences in structure between the domains are of crucial importance in the interaction with E1 and can also affect the reaction with lipoate protein ligase A (LplA), an enzyme responsible for the attachment of the lipoyl group to the target lysine residue (Morris et al., 1995; Green et al., 1995b).

\section{Results}

\section{Design of mutations}

In the structures of the E. coli E2p and E2o lipoyl domains (Figure 1), a prominent surface loop linking the first and second $\beta$-strands lies close in space to the exposed $\beta$-turn housing the lipoyllysine residue. Deletion of this loop from the lipoyl domain of B. stearothermophilus E2p renders the domain incapable of reductive acetylation by its partner E1p, while leaving it still capable of being lipoylated (Wallis et al., 1996). The surface loop is present in the $\beta$-sheet containing the lipoyl-lysine residue but not in the $\beta$-sheet across the 2 -fold axis of quasi-symmetry that contains the $\mathrm{N}$ and C-terminal residues. It was decided to make a series of mutations of this loop and test their effect on the post-translational modification and reductive acylation of the domain. The E2plip domain selected for these experiments was the innermost of the three in the E. coli E2p; the E2olip domain was the single one in the E2o chain.

First, the loop in the E. coli E2plip was replaced with the corresponding loop in the E. coli E2olip, to test whether this would shift the substrate specificity of the domain from E1p to E1o. The surface loop of the E2olip domain (residues 9 to 16) is two residues larger than its counterpart in E2plip (residues 9 to 14), as indicated in Figure 2, and the E2plip LS domain therefore has a loop two residues larger than the wild-type. To extend the loop replacement into the $\mathrm{N}$-terminal part of $\beta$-strand 2, additional mutations V15A and E16T were incorporated, to create the E2plip LS+ domain. The E2plip SAG domain was designed to have a loop sequence that is the same size as in the wild-type but lacks the charged side-chains of Asp13 and Glu14. The sequence chosen is loosely based on that of the surface loop in the A. vinelandii E2p lipoyl domains (Figure 2) and has a serine residue 
(a) Sequence alignment of the $\mathrm{N}$ - and $\mathrm{C}$ - terminal halves of various lipoyl domains

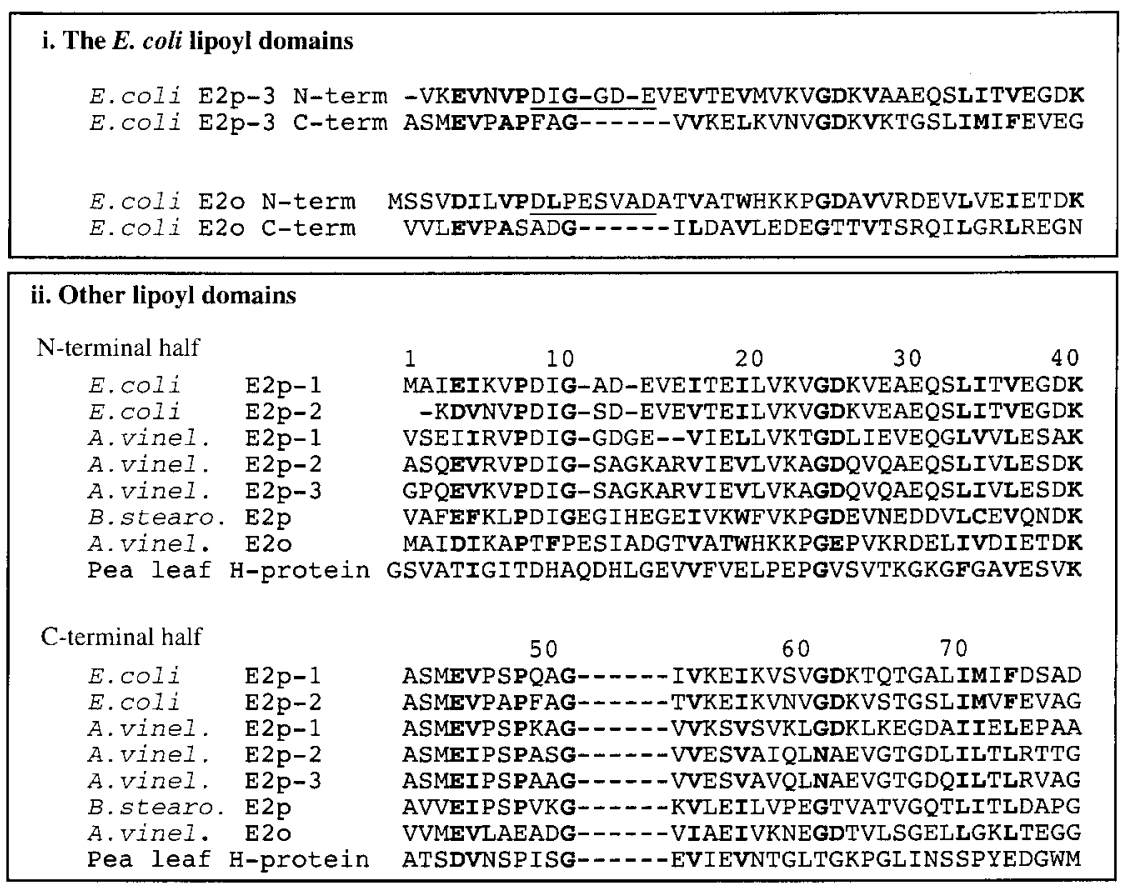

(b) Sequences of the wild-type (E2p-3) and mutant forms of the $E$. coli E2p lipoyl domain

\begin{tabular}{|c|c|c|}
\hline $\begin{array}{l}\text { Lipoyl } \\
\text { domain }\end{array}$ & $\mathrm{N}$-terminal sequence & $\begin{array}{l}\text { Lipoyl-lysine } \\
\beta \text {-turn region }\end{array}$ \\
\hline & $\begin{array}{lll}5 & 10 & 15\end{array}$ & 39 \\
\hline E2plip wt & VKEVNVPDIG-GD-EVEVT & EGDKAS \\
\hline E2plip LS & VKEVNVPDIPESVADVEVT & EGDKAS \\
\hline E2plip LS+ & VKEVNVPDIPESVADATVT & EGDKAS \\
\hline E2plip SAG & VKEVNVPDIGSAG--VEVT & EGDKAS \\
\hline E2plip G39T & VKEVNVPDIG-GD-EVEVT & ETDKAS \\
\hline E2plip G39T LS & VKEVNVPDIPESVADVEVT & ETDKAS \\
\hline E2plip LD & VKEVNVPDI ------ VEVT & EGDKAS \\
\hline E2olip wt & SSVDILVPDLPESVADATVA & ETDKVV \\
\hline
\end{tabular}

Figure 2. Sequence alignments of lipoyl domains and mutant forms of the E. coli E2p domain. (a) N and C-terminal halves of lipoyl domains from various sources. Identical and highly conserved residues are shown in bold. The numbering is that of the outermost of the three lipoyl domains (E2p-1) of E. coli E2p. The prominent surface loop between $\beta$-strands 1 and 2 is underlined in the sequences of the E. coli E2p-3 and E2o domains. The lipoylated lysine is K41. The pea leaf H-protein is included for comparison. (b) Sequence alignment of the wild-type (E2p-3) and mutant forms of the E. coli E2p lipoyl domain. Only the sequences of the N-terminal and lipoyl-lysine $\beta$-turn regions are indicated, where mutations were introduced. The numbering is the same as in (a).

to maintain a polar presence in the loop. Four residues (positions 11-14) were also deleted from the loop in the wild-type E2plip domain, thereby creating the E2plip LD domain.

The mutation N40A in the residue preceding the DKA motif in the lipoyl-lysine $\beta$-turn of the single lipoyl domain of B. stearothermophilus E2p severely inhibits the reductive acetylation of that lipoyl domain by its partner E1p (Wallis et al., 1996). In PDH complexes with multiple lipoyl domains in the E2p chain, the corresponding residue is conserved within a given E2p but varies between species and with the size of the surface loop. The side-chain at that location is either small and nonpolar or uncharged but polar and capable of forming hydrogen bonds. For example, in all three lipoyl domains of E. coli E2p, glycine is found at this position but is replaced by serine in each of the three lipoyl domains of $A$. vinelandii E2p (Figure 2). The corresponding residue in E. coli and A. vinelandii E2olip is a threonine (Thr42 in E. coli E2o). The mutation G39T was therefore introduced into the E. coli E2plip and E2plip LS domains to test its effect on the specificity of the domains as substrates for E1p and E1o. All the mutations are summarized in Figure 2. 


\section{Purification and characterization of the E2plip domains}

All the mutant domains could be purified from cell-free extracts of E. coli BL21(DE3) cells transformed with the relevant plasmid by the method used for the wild-type E2plip and E2olip domains (Dardel et al., 1990; Ricaud et al., 1996). The loopswap mutants were eluted from the Resource $Q^{\circledR}$ anion exchange column at a slightly higher concentration of ammonium bicarbonate than the wildtype protein (see Material and Methods), as expected given that they have a net charge of -1 more than the wild-type E2plip domain.

The E2plip domains were isolated in two main forms: with and without the N-terminal methionine residue (N-Met) in place. The extent of N-Met removal by methionyl aminopeptidase (Met-AP) depends in part on the neighbouring amino acid. Large, charged amino acids at position 2 lead to a lower efficiency of N-Met removal in E. coli (Hirel et al., 1989). In the E2plip domains used here, valine is the second amino acid and the N-Met-Val bond is normally cleaved quite efficiently (Hirel et al., 1989). The wild-type E2plip domain was recovered with just under $50 \%$ of the N-Met present, as judged by mass spectrometry, and the E2plip SAG domain behaved similarly. However, virtually all the N-Met was found to be removed from the E2plip LD domain, even though the neighbouring residue is still valine. In contrast, the E2olip domain has serine at position 2 and all the $\mathrm{N}$-Met was found to have been removed. Serine and valine as neighbouring amino acids have similar effects on processing efficiency (Hirel et al., 1989).

The large amount of lipoyl domain generated by over-expression of the relevant sub-gene from the pET11c plasmid may be swamping the Met-AP activity of the E. coli host cell, allowing only partial processing of N-Met, but the near-total processing of the E2plip LD and E2olip domains argues against this. Another possible explanation is that Met-AP activity is affected by the secondary structure of the substrate. If the second residue is part of a $\beta$-strand, as in the wild-type E2plip domain, the action of Met-AP could be hampered; if the first $\beta$-strand of the E2plip LD domain has not fully formed, removal of N-Met could proceed. In the E2olip domain, the natural $\mathrm{N}$ terminus of the E2o chain, $\beta$-strand 1 appears to start with Ser3 (Ricaud et al., 1996), and this might account for the efficient processing that it undergoes.

\section{Lipoylation of the domains in vitro}

Owing to the high level of expression of the subgenes in the pET11c plasmid system, all of the domains were isolated in the unlipoylated form, as indicated by electrospray mass spectrometry (Table 1). To prepare them as substrates for reductive acylation, the purified domains were therefore lipoylated in vitro using E. coli LplA. In view of the dependence of lipoylation on the structural integrity of the lipoyl domain (Reche et al., 1998; Reche \& Perham, 1999 and references therein), successful post-translational modification is a good indication that a domain has folded to a structure at least closely resembling that of the native protein. All the mutant domains other than E2plip LD were readily and fully lipoylated by this treatment, as judged by both mass spectrometry (Table 1) and nondenaturing PAGE (Figure 3). The E2plip LD mutant required a higher concentration of LplA and a longer incubation time to achieve full lipoylation, suggesting that it may be less well folded than the other mutant domains. As expected, all the lipoylated domains were eluted from a Resource $\mathrm{Q}^{\circledR}$ column at a higher salt concentration than their unlipoylated counterparts, owing to the loss of the positive charge on the target lysine residue that accompanies lipoylation.

As shown in Figure 3, the various domains were lipoylated by LplA at different rates. The wild-type E2plip domain was fully lipoylated within 90 minutes under the conditions used. In comparison, the E2plip LS domain was fully lipoylated within 30 minutes, over $60 \%$ of the domain being lipoylated within five minutes compared with only

Table 1. Molecular masses of the wild-type and mutant forms of the innermost lipoyl domain of E. coli E2p and of the lipoyl domain of E. coli E2o, as determined by positive-ion electrospray mass spectrometry

\begin{tabular}{|c|c|c|c|c|c|c|c|c|}
\hline \multirow[b]{3}{*}{ Lipoyl domain } & \multicolumn{4}{|c|}{ Mass of unlipoylated component (Da) } & \multicolumn{4}{|c|}{ Mass of lipoylated component (Da) } \\
\hline & \multicolumn{2}{|c|}{ Calculated } & \multicolumn{2}{|c|}{ Measured } & \multicolumn{2}{|c|}{ Calculated } & \multicolumn{2}{|c|}{ Measured } \\
\hline & - Met & + Met & - Met & + Met & - Met & + Met & - Met & + Met \\
\hline E2plip wt & 9107.6 & 9238.7 & $9107.0 \pm 0.4$ & $9238.5 \pm 1.1$ & 9295.9 & 9427.0 & $9297.1 \pm 0.6$ & $9425.7 \pm 1.0$ \\
\hline E2plip LS & 9345.9 & 9477.0 & $9346.5 \pm 0.9$ & $9477.0 \pm 0.2$ & 9534.2 & 9665.3 & $9534.5 \pm 0.3$ & $9665.6 \pm 0.9$ \\
\hline E2plip LS+ & 9289.6 & 9420.7 & $9291.2 \pm 0.6$ & $9418.9 \pm 1.2$ & 9477.9 & 9609.0 & $9476.3 \pm 0.1$ & $9608.4 \pm 0.9$ \\
\hline E2plip SAG & 9019.4 & 9151.5 & $9018.4 \pm 0.5$ & $9149.6 \pm 0.5$ & 9207.4 & 9338.5 & $9207.2 \pm 0.9$ & $9338.6 \pm 0.5$ \\
\hline E2plip G39T LS & 9390.0 & 9521.1 & $9390.9 \pm 0.8$ & $9522.0 \pm 1.1$ & 9578.3 & 9709.4 & $9577.6 \pm 1.1$ & $9709.2 \pm 1.1$ \\
\hline E2plip LD & 8747.2 & 8878.3 & $8746.57 \pm 0.6$ & $8877.0 \pm 0.3^{\mathbf{a}}$ & 8935.2 & 9066.3 & $8935.8 \pm 0.2$ & $\mathrm{~N} / \mathrm{D}$ \\
\hline E2plip wt & 9727.1 & 9859.2 & $9727.4 \pm 0.8$ & N/D & 9915.4 & 10047.5 & $9915.6 \pm 1.0$ & $\mathrm{~N} / \mathrm{D}$ \\
\hline
\end{tabular}

The wild-type E2plip domain and mutants were isolated in two forms, corresponding to species with the N-terminal methionine residue in place and those from which it had been removed. For the E2plip LD and wild-type E2olip domains, only a trace of the form with an N-Met residue was detected. N/D denotes not detectable.

a Denotes a minor peak. 


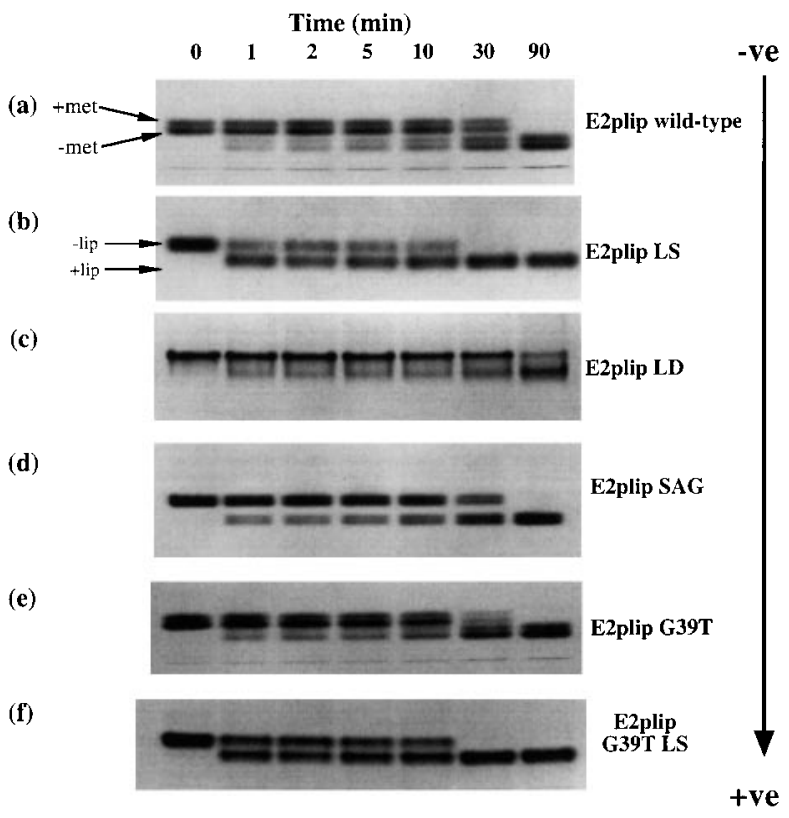

Figure 3. Lipoylation of the lipoyl domains with LplA in vitro. The apo-form of each lipoyl domain $(50 \mu \mathrm{M})$ was incubated with LplA under the conditions described in Materials and Methods. Samples were removed at various times and the products were analysed by means of non-denaturing PAGE (20\% gels). The gels were stained with Coomassie blue. Lipoylation of the E2plip wild-type (a), E2plip LS mutant (b), E2plip LD mutant (c), E2plip SAG mutant (d), E2plip G39T (e) and E2plip G39T LS mutant (f) domains. In each lane, the upper band represents the apo-form of the domain. For all the domains other than E2plip LD, each form appears as a doublet owing to the partial processing of the $\mathrm{N}$-terminal methionine residue.

ca $25 \%$ of the wild-type domain. The E2plip G39T mutant was lipoylated at a rate comparable with that of the wild-type whereas the E2plip G39T LS domain was lipoylated more rapidly, at a rate similar to that of the E2plip LS domain. Although the rate of lipoylation of the E2p LD domain was greatly reduced, the fact that it could be lipoylated at all means that it must have acquired some degree of structure. Sequence motifs (DKA or DKV) from the lipoyl-lysine $\beta$-turn are present elsewhere in the E2plip domain but only the correct lysine residue was lipoylated; presentation of the target lysine residue at the tip of a properly constituted $\beta$-turn in a folded domain is known to be essential for correct post-translational modification (Wallis \& Perham, 1994).

\section{Reductive acylation of E2plip LS domains}

All the mutant lipoyl domains with an interchanged surface loop (Figure 2) were assayed for their ability to become reductively acetylated (by $\mathrm{E} 1 \mathrm{p}$, in the presence of pyruvate) and succinylated (by E1o, in the presence of 2-oxoglutarate). Replacement of four residues in the E2plip loop with six of those of the E2olip loop (E2plip LS) and the additional replacement of Val15 and Glu16 in the second $\beta$-strand (E2plip LS+) led to much lower rates of reductive acetylation of the mutant domains in the presence of E1p and pyruvate (Figure 4(a)). The extent of acetylation after $25 \mathrm{~min}$ utes was also lower than that of the wild-type E2plip domain (Figure 4b). Thus, altering the size and character of the surface loop did not prevent the E2plip domain from being reductively acetylated by E1p but the rate of acetylation was affected; the loop between $\beta$-strands 1 and 2 is evidently not the sole determinant of successful interaction. These effects could be due to the increase in the size of the loop, rather than the loss of a particular protein-protein interaction. The decrease in the extent of reductive acetylation would suggest that the stability of the thioester bond linking the acetyl group to the lipoyl moiety has also been affected. Residues Val15 and Glu16 appear to be unimportant for domain recognition, as the E2plip LS+ domain behaved in a similar way to the E2plip LS domain.

The rate and overall extent of reductive succinylation of the E2plip LS and E2plip LS+ domains in the presence of 2-oxoglutarate and E1o were both found to be low, little different from those of the wild-type E2plip domain (Figure 5(a) and (b)). Thus, exchanging surface loops between the E2plip and E2olip domains has also not increased the ability of the E2plip domain to function as a substrate for E1o.

\section{Reductive acylation of the E2plip G39T domains}

In the reductive acetylation assay, the E2plip G39T domain (Figure 2) was virtually indistinguishable from the wild-type domain (Figure 4(c) and (d)). This is in marked contrast with the equivalent mutation $(\mathrm{N} 40 \mathrm{~A})$ in the B. stearothermophilus E2p lipoyl domain, which virtually eliminated its ability to be reductively acetylated by its partner E1p (Wallis et al., 1996). When the G39T mutation was incorporated into the E2plip LS domain, the rate (Figure 4(c)) and overall extent (Figure 4(d)) of reductive acetylation were very similar to those of the E2plip LS domain itself (Figure 4(a) and (b)). The G39T mutation therefore appears to have had little or no additional effect, the LS mutation rendering the E2plip domain able to interact with E1p much less effectively, as before.

Reductive succinylation of the G39T mutants was also tested to see if the domains had gained in ability to be recognized by E1o. As shown in Figure 5(c), the rate of reductive succinylation was very low, close to that of the wild-type E2plip domain. Likewise, the overall extent of succinylation of both the E2plip G39T and E2plip G39T LS domains after 25 minutes was only a little above that of the E2plip domain and still way below that of the wild-type E2o domain (Figure 5(d)). Thus, for the E.coli E2p lipoyl domains, the residue 
a.

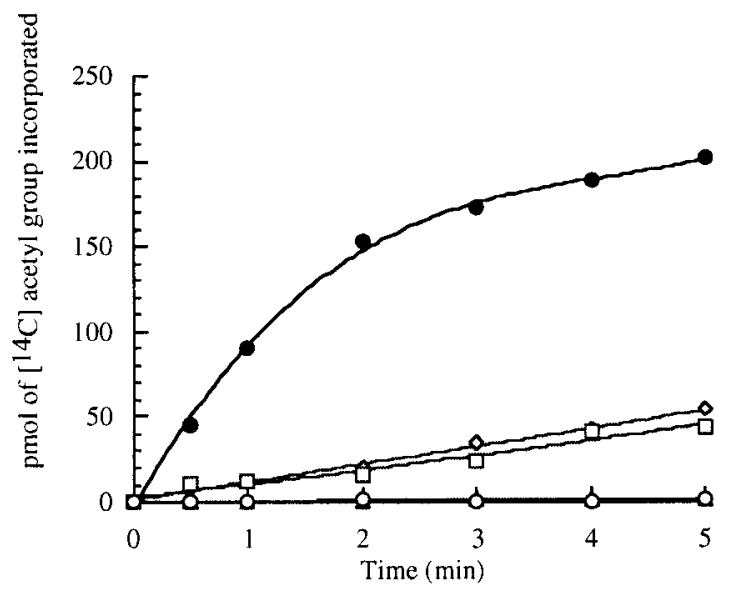

c.

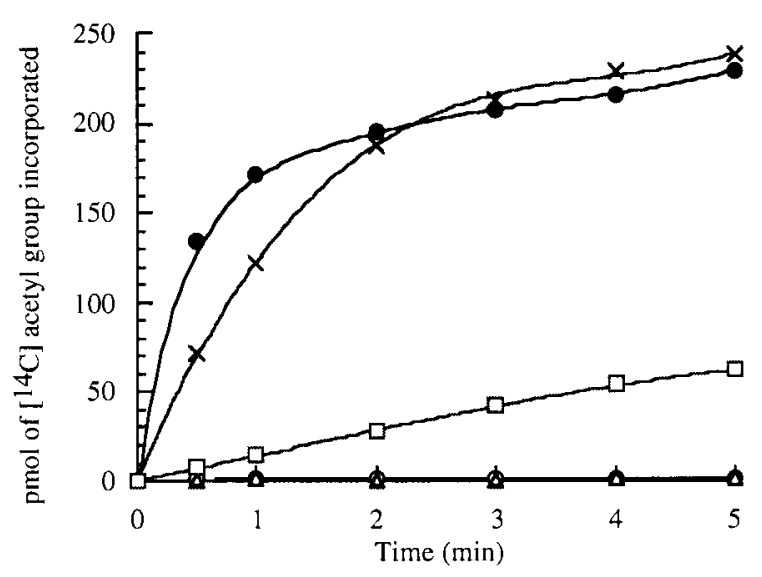

e.

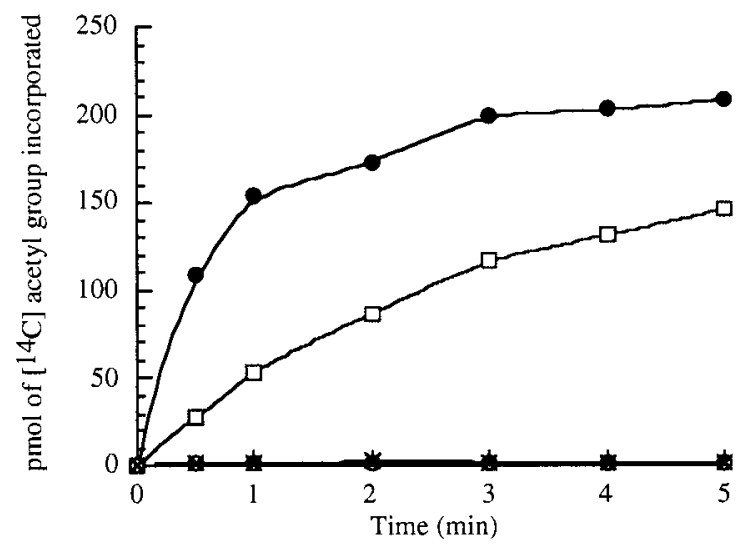

b.

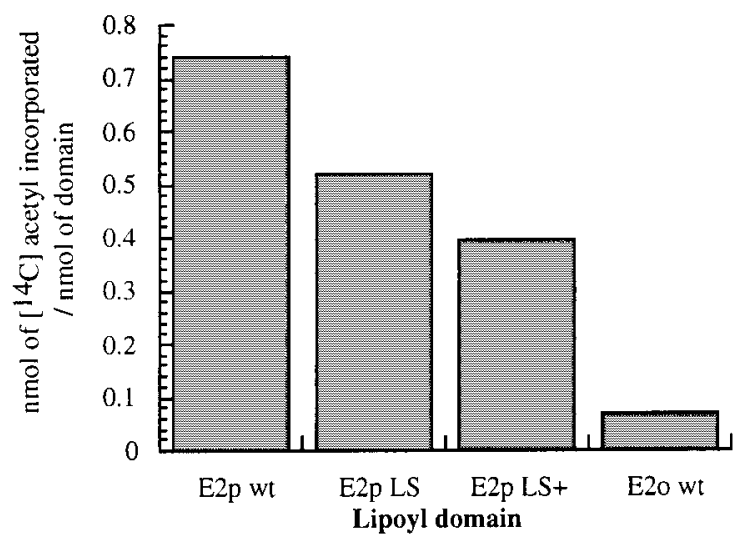

d.

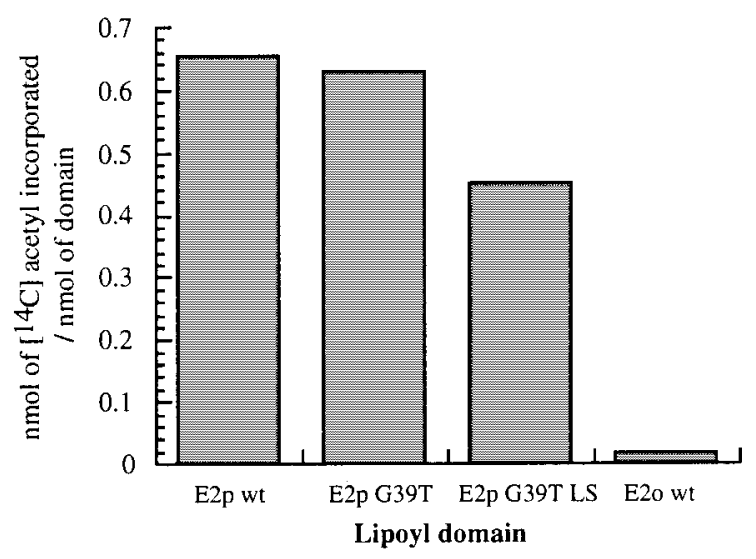

f.

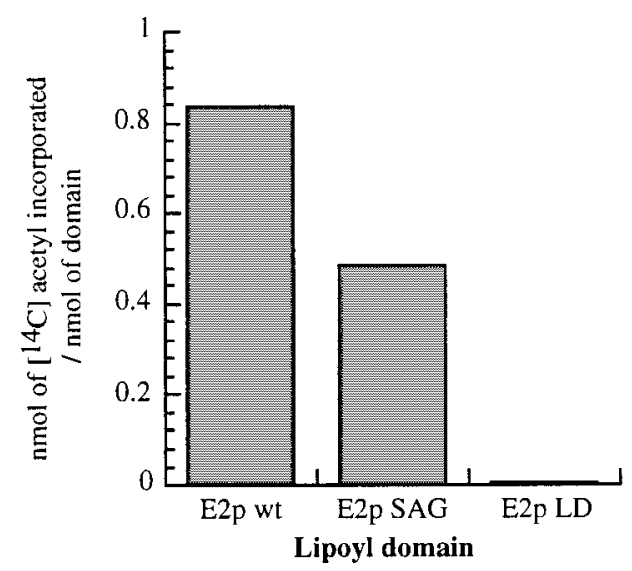

Figure 4. Reductive acetylation of the wild-type and mutant lipoyl domains by E1p in the presence of pyruvate. The domain was incubated with E1p and [2- $\left.{ }^{14} \mathrm{C}\right]$ pyruvate as described in Materials and Methods and the radioactivity incorporated was measured at various times. (a) E2plip LS and LS+ domains and E2olip domain. (O) E2plip wt; $(\diamond)$ E2plip LS; $(\square)$ E2plip LS + ; $\triangle$ ) E2olip domain; $(\bigcirc)$ blank. (b) E2plip LS and LS+ domains and E2o domain. Extent of acetylation after 25 minutes. (c) E2plip G39T and G39T LS domains. (O) E2plip wt; $(\times)$ E2plip G39T; $(\square)$ E2plip G39T LS; $(\triangle)$ E2olip domain; $(\bigcirc)$ blank. (d) E2plip G39T and G39T LS domains. Extent of acetylation after 25 minutes. (e) E2plip SAG and E2plip LD domains. (O) E2plip wt; ( $\square$ ) E2plip SAG; (×) E2plip LD; (O) blank. (f) E2plip SAG and E2plip LD domains. Extent of acetylation after 25 minutes. 
a.

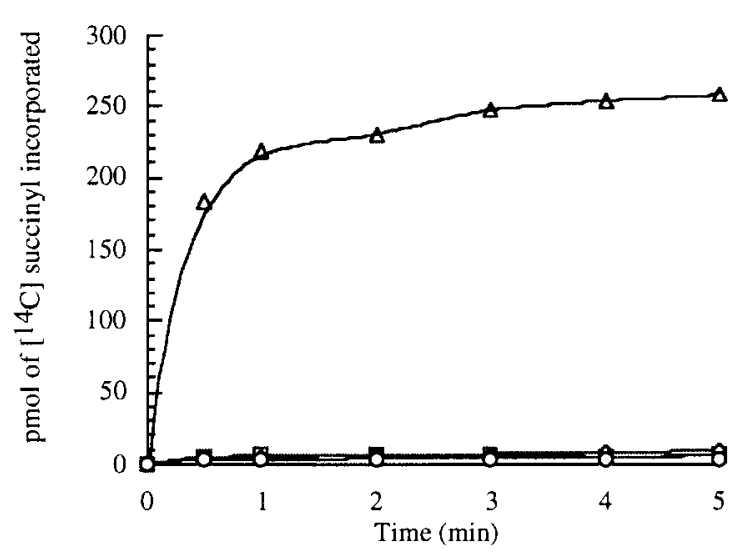

c.

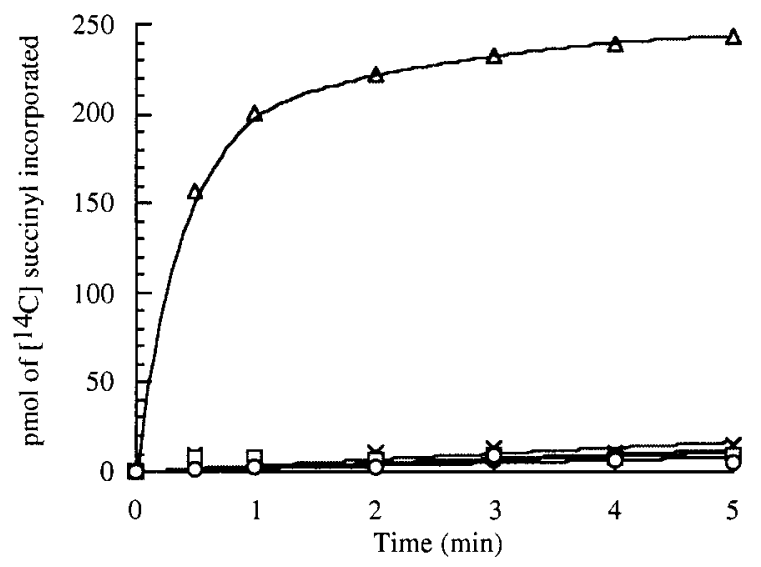

b.

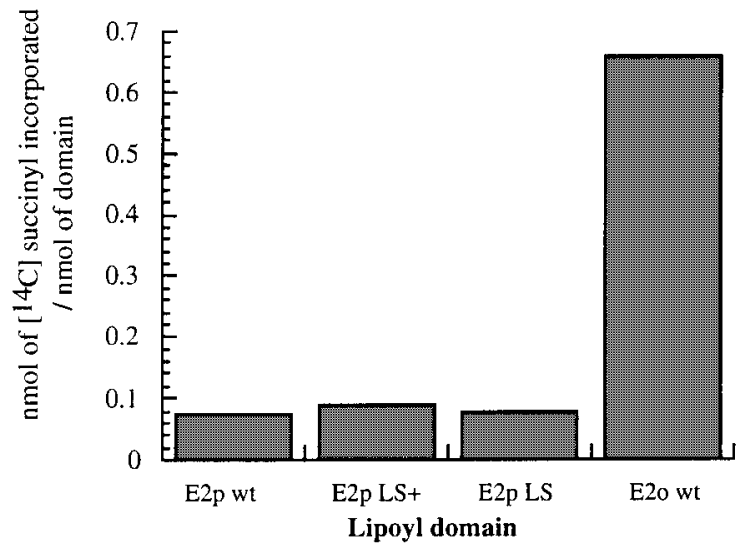

d.

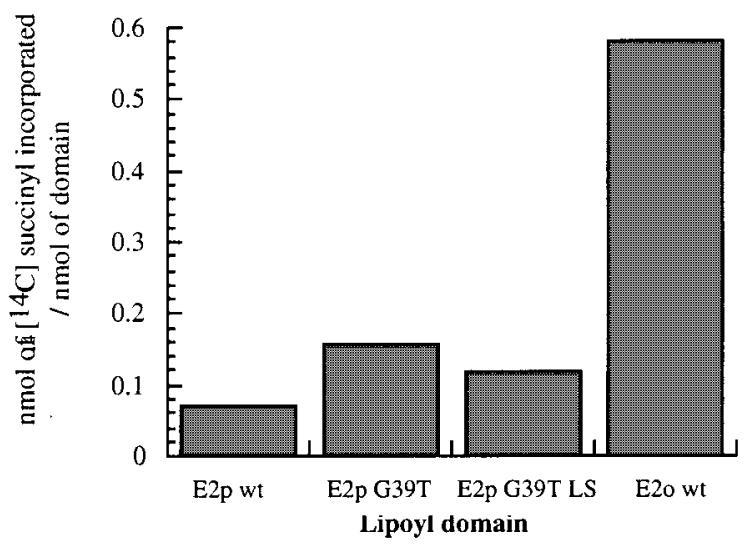

Figure 5. Reductive succinylation of the wild-type and mutant lipoyl domains by E1o in the presence of 2-oxogluta-

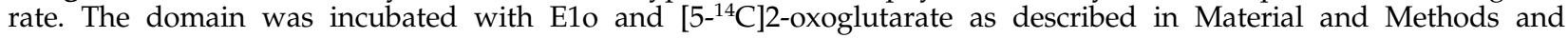
the radioactivity incorporated was measured at various times. (a) E2plip LS and LS+ domains and E2plip domain. $(\triangle)$ E2olip wt; $(\diamond)$ E2plip LS; $(\square)$ E2plip LS + ; (○) E2plip domain; (○) blank. (b) E2plip LS and LS+ domains and E2plip domain. Extent of succinylation after 25 minutes. (c) E2plip G39T and G39T LS domains. $(\triangle)$ E2olip wt; (×) E2plip G39T; ( $\square$ ) E2plip G39T LS; (O) E2plip domain; (O) blank. (d) E2plip G39T and G39T LS domains. Extent of succinylation after 25 minutes.

immediately preceding the $\beta$-turn housing the lipoyl-lysine residue does not engage in specifying the interaction between the lipoyl domain and E1p, although it may play a small part in the recognition of the lipoyl domain by E1o.

\section{Reductive acetylation of the E2plip SAG domain}

The E2plip SAG domain has a loop the same size as that in the wild-type but differing in sequence (Figure 2). As shown in Figure 4(e), the rate of reductive acetylation of the E2plip SAG domain by E1p was below that of the wild-type but considerably above that of the LS mutants (Figure 4(a)). On this basis, it was expected that the
E2plip SAG domain would reach the same level of reductive acetylation after 25 minutes as the wildtype E2plip domain, but it achieved only ca $60 \%$ of this value (Figure 4(f)). As with the LS mutants (see above), it would appear that changes to the surface loop influence the interaction with E1p and affect the stability of the thioester bond that links the acetyl group to the dihydrolipoyl moiety of the E. coli E2p lipoyl domain.

\section{Reductive acetylation of the E2plip LD domain}

Deletion of four residues involved in the surface loop linking $\beta$-strands 1 and 2 (Figure 2) left the E2plip domain incapable of becoming reductively acetylated (Figure 4(e) and (f)), as was found pre- 
viously for the B. stearothermophilus E2p lipoyl domain (Wallis et al., 1996). However, in addition to being inactive in the reductive acetylation assay, the E. coli E2plip LD domain was a relatively poor substrate for lipoylation by LplA (Figure 3), strongly suggesting that deletion of the loop may have caused some disruption of the tertiary structure.

\section{Folding of the mutant domains}

Two of the mutant domains, E2plip LS and E2plip LD, were selected for further analysis by means of NMR spectroscopy, to investigate their folding. The 1D spectra of the E2plip (wild-type), E2plip LS and E2plip LD domains, all unlipoylated, are shown in Figure 6(a). It is clear from the poor dispersion of peaks in the $\mathrm{H}^{\mathrm{N}}$ region, in comparison with the wild-type, that the E2plip LD domain is partially unfolded. Similarly, the number of $\mathrm{H}^{\alpha}$-peaks downfield of the water signal has decreased, indicating the loss of some secondary structure (Figure 6(b)). However, there is evidence that part of the hydrophobic core may be intact (Figure 6(c)). The peaks upfield and slightly downfield of the TSP signal $(-0.4 \mathrm{ppm}$ to $+0.4 \mathrm{ppm})$ are due to the $\mathrm{C}^{\gamma}$ protons $\left(\mathrm{H}^{\gamma / \#}\right)$ of Val5 (D.D.J., unpublished work). The reason for their unusual chemical shift is their proximity to an aromatic ring system involving Phe50 and Phe74, the latter forming part of the hydrophobic core in the wild-type domain (Green et al., 1995a; D.D.J., unpublished work); their position in the spectrum of the E2plip LD domain implies that it is folded to some degree. The 2D NOESY spectrum of the E2plip LD domain (Figure 7) gave additional information. The lack of dispersion in the $\mathrm{H}^{\mathrm{N}}-\mathrm{H}^{\alpha}$ region and the loss of cross-peaks in the $\mathrm{H}^{\mathrm{N}}-\mathrm{H}^{\mathrm{N}}$ proton region (evidence for turns in the structure) again indicated that the protein was not fully folded. A limited structuring of the E2plip LD domain would account for its lower rate of lipoylation by LplA (Figure 3) and the subsequent inability to be reductively acetylated (Figure 4).

In similar experiments, the E2plip LS domain showed a well-dispersed $\mathrm{H}^{\mathrm{N}}$ region and retained all the $\mathrm{H}^{\alpha}$-peaks downfield of water indicative of extensive $\beta$-sheet structure. Further, in 2D NMR spectra, the proton chemical shifts showed a high degree of similarity to those of the wild-type E2plip domain (data not shown). It can therefore be concluded that the E2plip LS domain was correctly folded and had a native-like structure. This is consistent with the fact that the mutant domain was rapidly lipoylated (Figure 3) and could be reductively acetylated, albeit at a significantly lower rate than wild-type, by E1p (Figure 4).

\section{Formation of the lipoyl-lysine $\beta$-turn in the E2plip LD domain}

For lipoylation of the lipoyl domain to take place, the target lysine has to be positioned cor- rectly in a protruding $\beta$-turn; shifting the lysine residue one place to the $\mathrm{N}$ or $\mathrm{C}$-terminal side results in no post-translational modification (Wallis \& Perham, 1994). Disruption of this region could well have a deleterious effect on lipoylation. Further analysis of the 2D NOESY spectra of the E2plip LD domain revealed NOE cross-peaks that could imply a degree of structure in the lipoyllysine region. As shown in Figure 8(a), long-range cross $\beta$-strand $\mathrm{H}^{\alpha}-\mathrm{H}^{\alpha}$ NOEs could be detected for residues constituting $\beta$-strands 4 and 5 , which form the lipoyl-lysine $\beta$-turn. The E2plip LD mutant retained the tentatively assigned Glu38:Ser43 NOE but lacked the Glu16:Lys64 and Glu45:Thr36 NOE cross-peaks observed for the wild-type E2plip domain. The presence of the Glu38:Ser43 NOE could mean that the two anti-parallel $\beta$-strands constituting the $\beta$-turn were partially formed but the loss of the Glu45:Thr36 NOE suggests that this was not as extensive as in the wild-type.

$\mathrm{H}^{\mathrm{N}}-\mathrm{H}^{\mathrm{N}}$ NOEs from the lipoyl-lysine (Lys41) and Ala42 define the $\beta$-turn. The wild-type E2plip and E2plip LS domains had strong Lys41:Ala42 NOEs (Figure 8(b)), indicating that the amide protons were within $2.5 \AA$ of each other. The Ala42:Gly39 $\mathrm{NOE}$, another important indicator of the $\beta$-turn, was present in the spectra of the E2plip LS domain and wild-type domains. However, neither of these NOEs was observed in the same region for the E2plip LD domain (Figure 8(b)). Cross-peaks corresponding to the Lys41:Ala42 and Ala42:Gly39 NOEs have been assigned only speculatively for the E2plip LD mutant but are consistent with both the wild-type and E2plip LS NOE characteristics. Their chemical shifts are very different from those of the wild-type E2plip and E2plip LS domains. Most of the $\mathrm{H}^{\mathrm{N}}-\mathrm{H}^{\mathrm{N}}$ NOEs still present are thought to be from that half of the lipoyl domain across the 2 -fold axis from the surface loop and lipoyl-lysine $\beta$-turn (see Figure 1).

Additional evidence for the partial folding of the E2plip LD domain comes from the $\mathrm{H}^{\alpha}-\mathrm{H}^{\mathrm{N}}$ region of the NOESY spectrum. As stated previously, several peaks in the $\mathrm{H}^{\alpha}$-region downfield of the water were missing from the 1D spectrum (Figure 6). Detailed analysis of the 2D NOESY spectrum revealed that intra- and inter-residue NOE crosspeaks had disappeared (Figure 9). With respect to the formation of the lipoyl-lysine $\beta$-turn, NOE cross-peaks involving Ile35, Thr36, Val37 and Glu45 had experienced a substantial change in chemical shift and were no longer present in this region. Other residues that underwent a significant change in chemical shift while remaining in the same region are Glu38, Gly39 and Ser43, all three close in space to the lipoyl-lysine residue.

\section{Formation of the $\mathrm{N}$-terminal $\beta$-strand and relationship to $\mathrm{N}$-Met processing}

In the $\mathrm{H}^{\alpha}-\mathrm{H}^{\mathrm{N}}$ region, the NOEs corresponding to Val2 and Asn6 are also missing from the spectrum 
a.

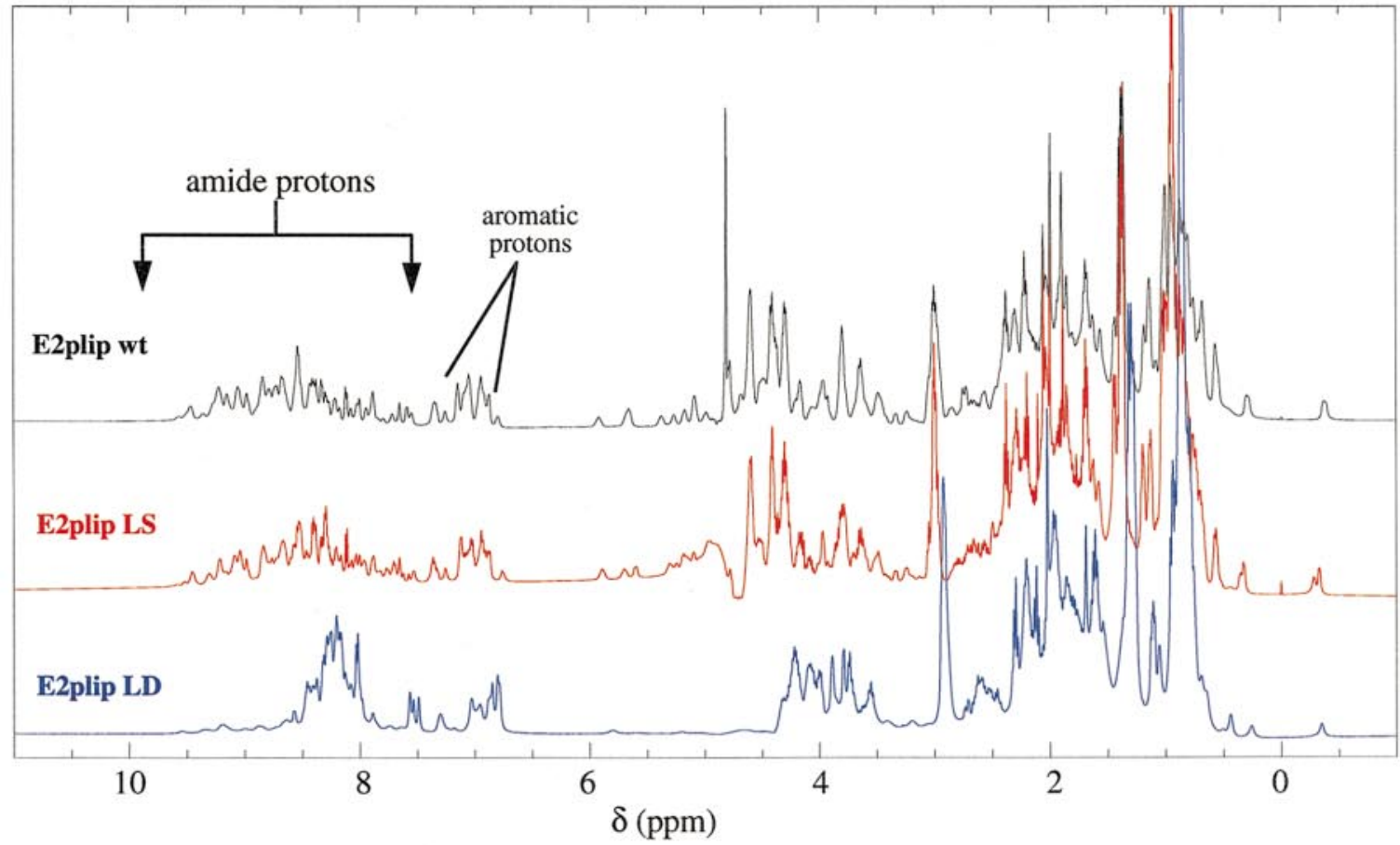

b.

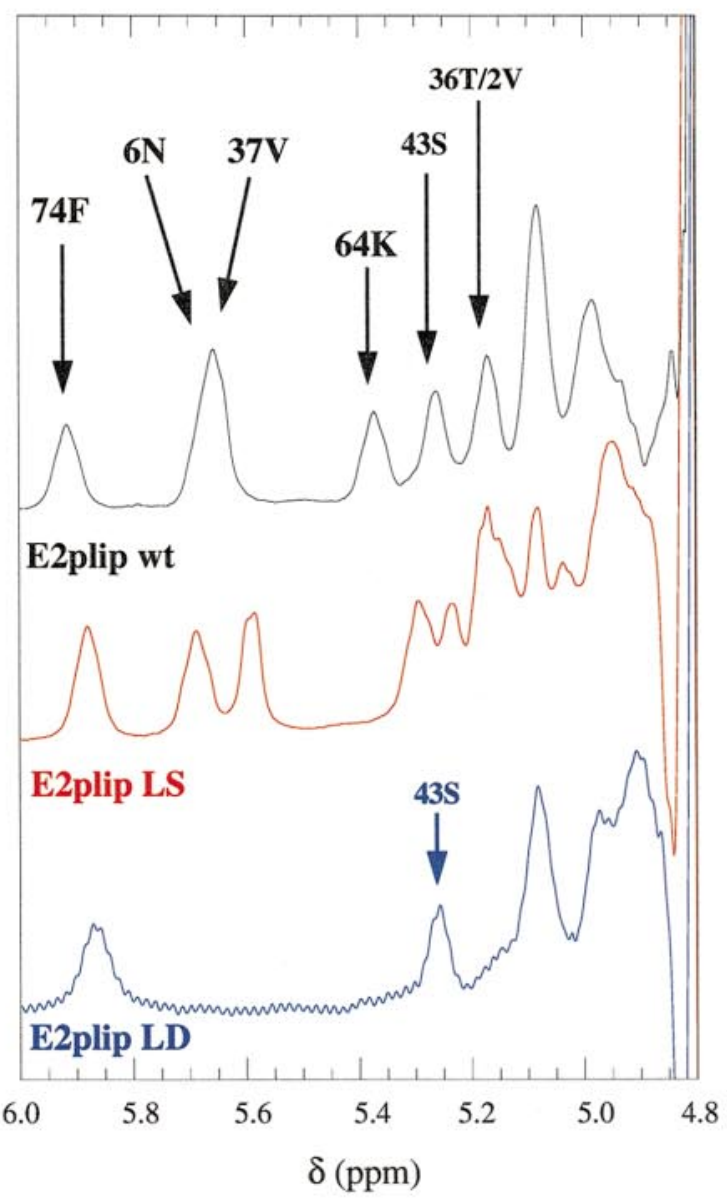

c.

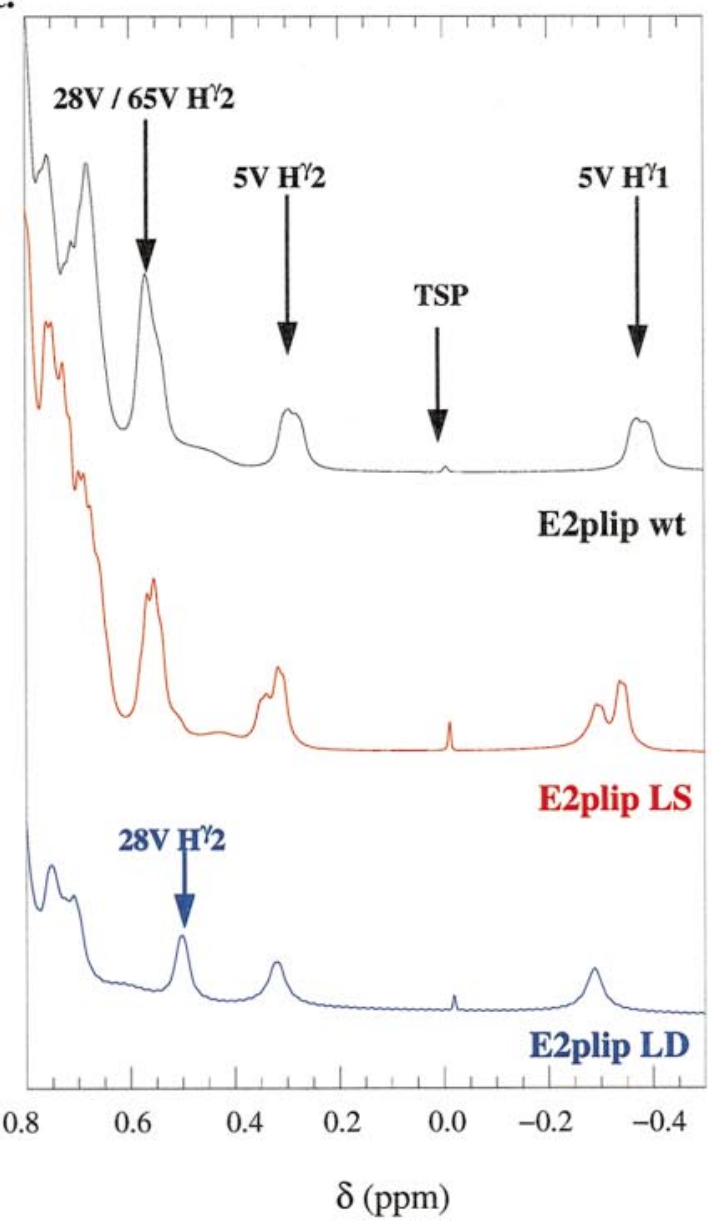

Figure 6. 1D NMR spectra of the wild-type and mutant lipoyl domains. Wild-type E2plip domain (black), E2plip LS domain (red), and E2plip LD domain (blue). The spectra of the domains (2-3 mM) in $20 \mathrm{mM}$ sodium phosphate buffer ( $\mathrm{pH}$ 6.0), were recorded at $298 \mathrm{~K}$, with trimethylsilyl propionate (TSP) as the internal reference. (a) The complete spectrum, (b) $\mathrm{H}^{\alpha}$ peaks downfield of the water that are indicative of $\beta$-sheet formation, (c) $\mathrm{H}^{\gamma} \#$ peaks of Val5 between -0.4 and $+0.4 \mathrm{ppm}$. 


\section{a. $\mathbf{H}^{\alpha}-\mathbf{H}^{\mathrm{N}}$ region}

(i) E2plip wt

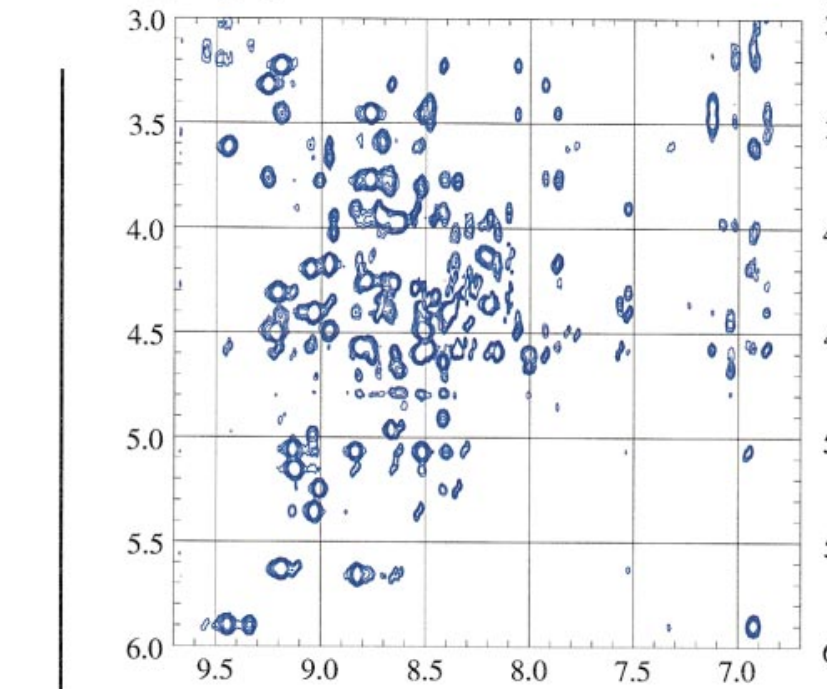

(ii) E2plip LD

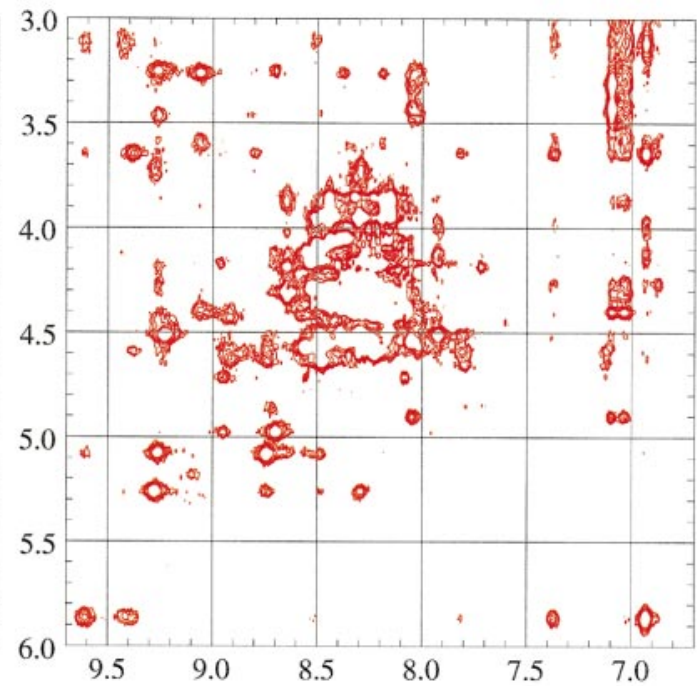

츨

b. $\mathrm{H}^{\mathrm{N}}-\mathrm{H}^{\mathrm{N}}$ region

(i) E2plip wt

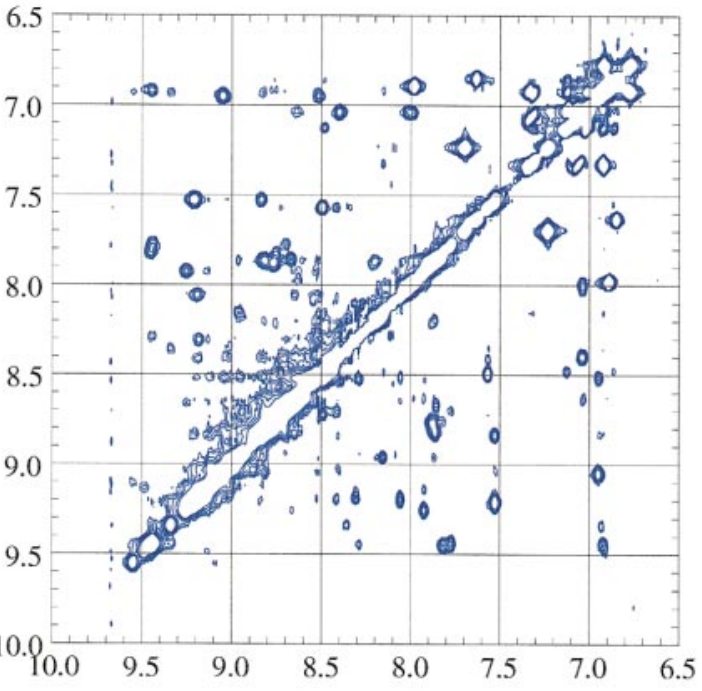

(ii) E2plip LD

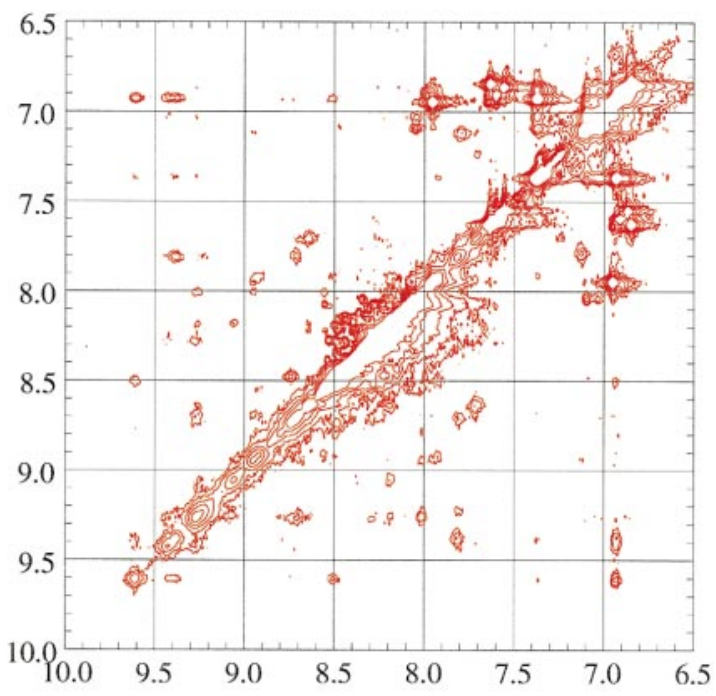

\section{$\delta \mathbf{H}(\mathrm{ppm})$}

Figure 7. 2D NOESY spectra of the wild-type and E2plip LD lipoyl domain. (a) Fingerprint $\mathrm{H}^{\alpha}-\mathrm{H}^{\mathrm{N}}$ region, (b) interresidue $\mathrm{H}^{\mathrm{N}}-\mathrm{H}^{\mathrm{N}}$ region. Cross-peaks between 6.5 and $7.5 \mathrm{ppm}$ correspond to intra-residue NOEs from the aromatic side-chains of Phe50 and Phe74. All NOESY spectra were recorded at $298 \mathrm{~K}$ and pH 6.0.

of the E2plip LD domain (Figure 9). This implies that they may no longer be in the original $\beta$-strand conformation and supports the view that a disruption of the secondary structure in the N-terminal region may facilitate Met-AP activity, permitting a nearly complete removal of N-Met from the E2plip LD domain (see above).

\section{Discussion}

Specific post-translational modification and reductive acylation are essential properties of the lipoyl domains of 2-oxo acid dehydrogenase complexes. Lipoylation of the correct lysine residue and the subsequent reductive acylation of the lipoyl group by the cognate E1 component are 


\section{a. $\mathbf{H}^{\alpha}-\mathrm{H}^{\alpha}$ NOEs}
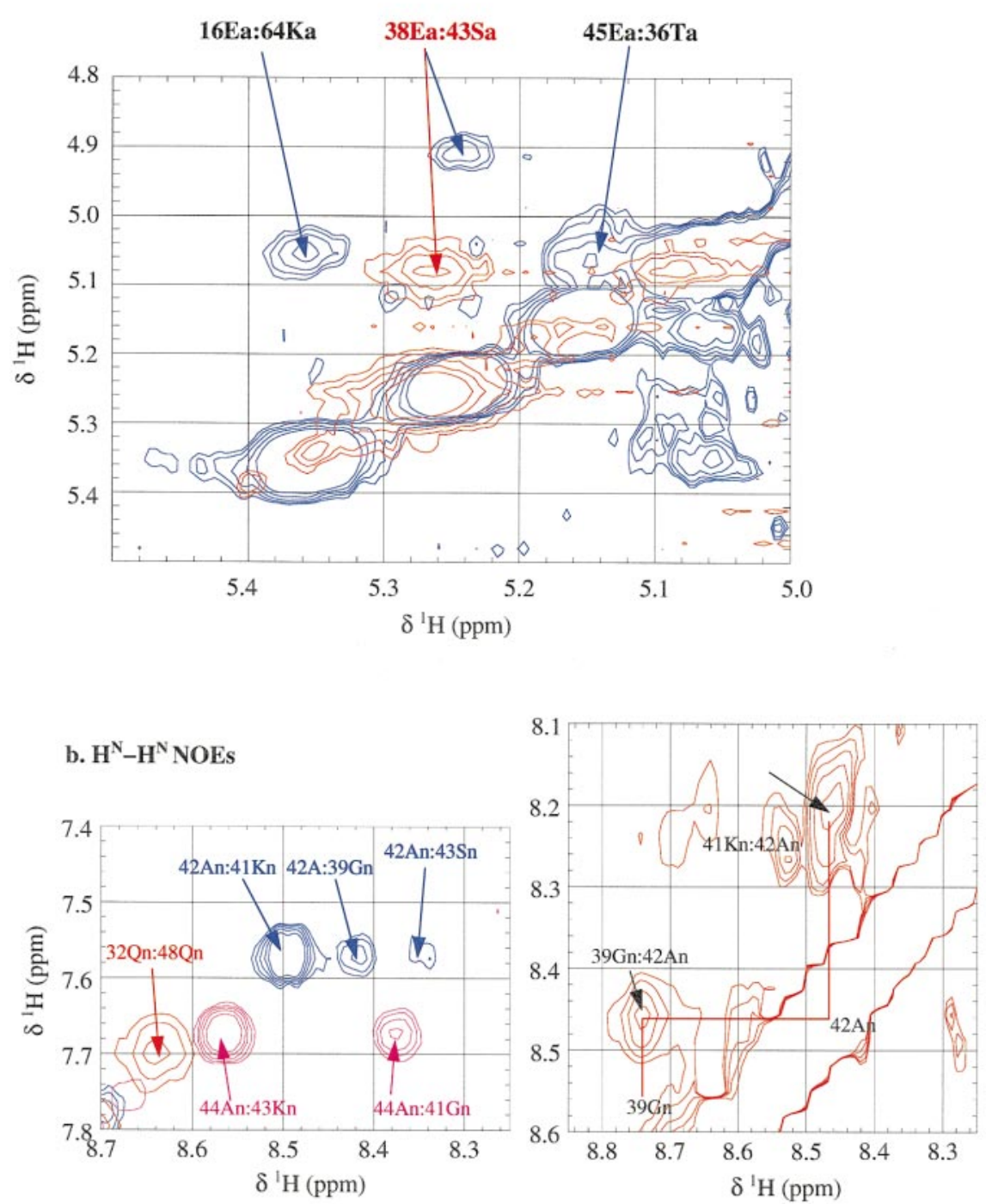

Key:

$\mathrm{Xa}=\mathrm{H}^{\alpha}$

$\mathrm{Xn}=\mathrm{H}^{\mathrm{N}}$

Figure 8. Evidence for the formation of the lipoyl-lysine $\beta$-turn in the E2plip LD domain. (a) Cross-strand $\mathrm{H}^{\alpha}-\mathrm{H}^{\alpha}$ NOEs for residues in $\beta$-strands 4 and 5 , (b) $\mathrm{H}^{\mathrm{N}}-\mathrm{H}^{\mathrm{N}}$ NOEs that define the lipoyl-lysine $\beta$-turn. The NOE cross-peaks for the E2plip LD domain have been tentatively assigned. The E2p wt spectrum is shown in blue, the E2p LS spectrum is purple and the E2p LD spectrum is red. All NOESY spectra were recorded at $298 \mathrm{~K}$ and $\mathrm{pH} 6.0$.

both governed by precise protein-protein interactions between the lipoyl domain and the relevant enzyme. Without a structured lipoyl domain, neither event will occur or will do so at a much reduced rate (Graham et al., 1989; Wallis et al., 1996; Berg et al., 1998; Reche et al., 1998; and references therein).

Lipoylation by LplA is less specific than reductive acylation by E1. LplA will lipoylate the E2o and E2p lipoyl domains of E. coli, as well as lipoyl domains from other species (Quinn et al., 1993; Wallis \& Perham, 1994; Macherel et al., 1996; Berg et al., 1998; K. Tozawa, unpublished work), and these lipoyl domains must have features in common for this to occur. The correct positioning of the target lysine residue in the exposed $\beta$-turn is one such determinant (Wallis \& Perham, 1994). We have shown here that exchanging the surface loop sequence of the E2plip domain (four residues) for that of the E2olip domain (six residues), increased the rate at which the E2plip LS domain was lipoylated (Figure 3). In contrast, the G39T mutation, characteristic of the E2o domain in the lipoyl-lysine $\beta$-turn, had no effect on the lipoylation rate of 


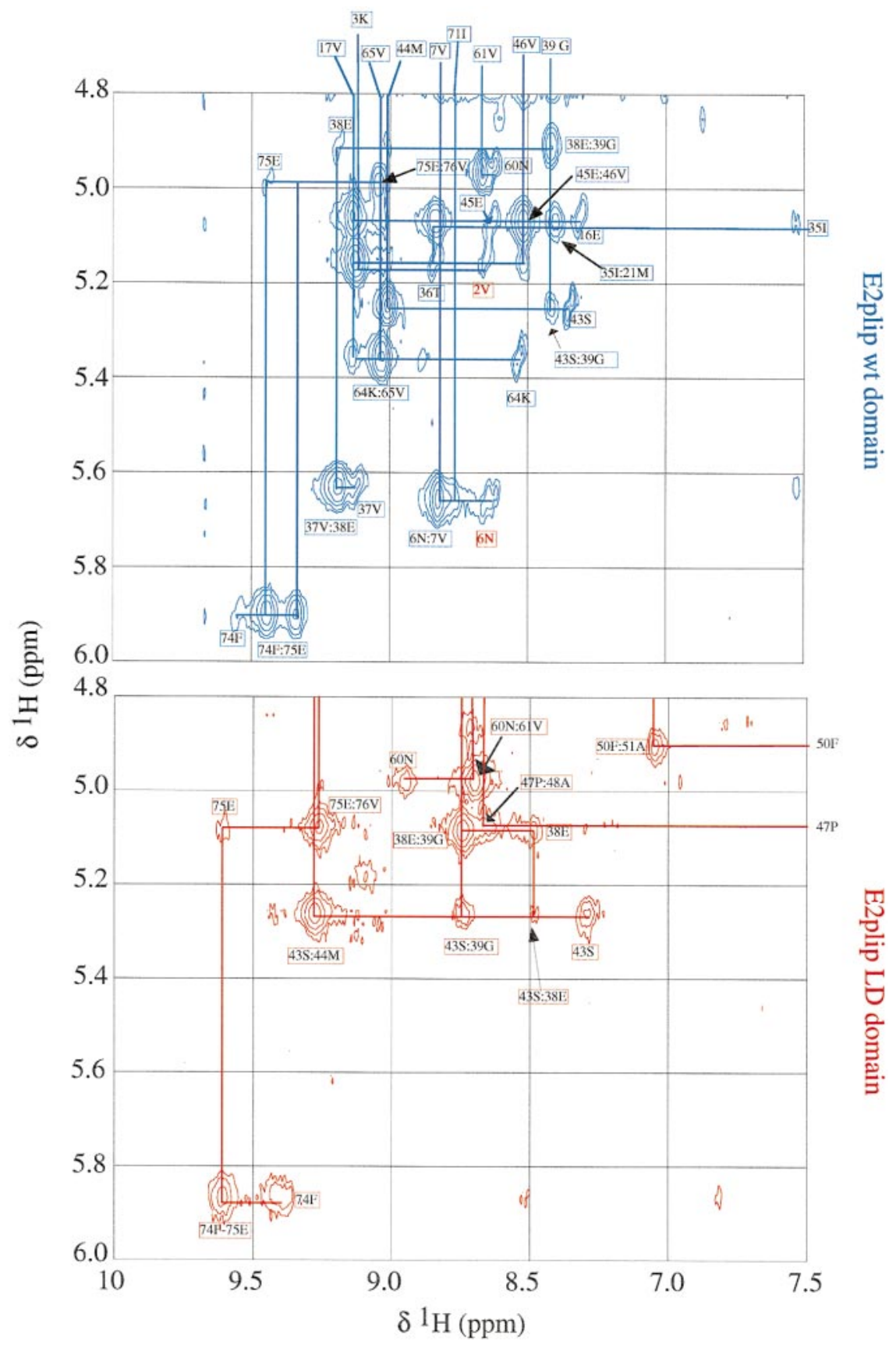

Figure 9. Comparison of the $\mathrm{H}^{\alpha}-\mathrm{H}^{\mathrm{N}}$ region from the NOESY spectra of the wild-type and E2plip LD lipoyl domains. (a) E2plip wt domain, (b) E2plip LD domain. Major changes have occurred upon deletion of four residues from the surface loop, notably the loss of cross-peaks from Ile35, Thr36, Val37 and Glu45 from this part of the spectrum of the E2plip LD domain. Other residues that are close to the lipoyl-lysine and still present in this area of the spectrum of the E2plip LD domain undergo significant changes in chemical shift (Glu38, Gly39, Ser43 and Met44). The cross-peaks highlighted in red in (a) are those corresponding to residues in the N-terminal $\beta$-strand not visible in (b).

E2plip or the E2plip LS domain (Figure 3). Moreover, the E2plip SAG domain was lipoylated at a rate comparable with that of the wild-type domain (Figure 3). Thus the exposed surface loop is not a major determinant of lipoylation, nor is the residue preceding the DKA motif in the lipoyl-lysine $\beta$ turn. As observed elsewhere (Wallis \& Perham,
1994; Reche et al., 1998), sequence changes in the DKA motif itself are also tolerated by LplA. More detailed structural studies of the E2plip LS domain suggest that the larger, variant loop has pulled away slightly from the lipoyl-lysine $\beta$-turn, compared with the position of the corresponding loop in both the wild-type E2p (D.D.J., unpublished 
work) and E2o (Ricaud et al., 1996) domains. This might allow for easier access to the target lysine residue by LplA.

In contrast, deletion of the surface loop led to a much reduced rate of lipoylation (E2plip LD domain; Figure 3). Loop deletion was accompanied by a partial loss of structural integrity on the part of the domain, as judged by NMR spectroscopy (Figures 6, 7 and 9), suggesting this as the probable reason for the impaired lipoylation. The $\beta$-turn has to be formed in order to present the target lysine residue correctly to LplA, and the NMR data described above suggest that the anti-parallel $\beta$ sheets 4 and 5 may not have been correctly formed in the E2plip LD domain. However, it is clear that LplA does not require a fully folded domain as its substrate, providing certain structural features are retained.

Reductive acylation of a lipoyl domain by its cognate E1 is far more demanding than lipoylation by LplA. The E2plip domain is recognised only by E1p and the E2olip domain only by E1o (Graham et al., 1989; and Figures 4 and 5 above). The specificity of these protein-protein interactions is essential for the precise substrate channelling that occurs within the complexes (Perham, 1991). As the two lipoyl domains share a virtually identical folding pattern (Green et al., 1995a; Ricaud et al., 1996; D.D.J., unpublished work), subtle differences must be responsible for instilling specificity, and the prominent surface loop linking the first and second $\beta$-strands has been implicated in this for the B. stearothermophilus PDH complex (Wallis et al., 1996). Reductive acetylation of the E. coli E2plip LS and E2plip LS+ domains by E1p was found still to occur but the rate was substantially lower than that of the wild-type E2plip domain (Figure 4). This could be due to the increase in size of two residues of the loop in the modified domain, thereby generating steric hindrance to the interaction with $\mathrm{E} 1 \mathrm{p}$, rather than alteration of a precise feature in the recognition of one protein by the other. Equally, the loop exchange did not lead to a significant increase in reductive succinylation of the E2plip domain by E1o (LS and LS+ domains; Figure 5). Berg et al. (1998) have also reported that importing the loops from the E2p domains into the E2o domain of $A$ vinelandii does not markedly improve it as a substrate for A. vinelandii E1p. Both the susceptibility to lipoylation (Figure 3) and 1D (Figure 6) and 2D (data not shown) NMR spectroscopy indicate that the E. coli E2plip LS domain has a native-like structure and that an additional two amino acids in the loop have been accommodated without effect. Simple replacement of the charged residues (GDE $\rightarrow$ SAG) in the loop in the E2plip SAG domain also affected the rate of the reductive acetylation (Figure 4) but not to the extent of the E2plip LS or E2plip LS+ domains. On this evidence, the surface loop is not uniquely responsible for the specificity of the recognition by $\mathrm{E} 1$, but does play a role either by means of a direct interaction or by maintaining the domain in a conformation required for recognition.

The replacement of asparagine with alanine $(\mathrm{N} 40 \mathrm{~A})$ on the N-terminal side of the DKA motif in the lipoyl-lysine $\beta$-turn of the lipoyl domain from the B. stearothermophilus PDH complex severely inhibits reductive acetylation by its E1p (Wallis et al., 1996). In contrast, replacement of the corresponding glycine residue (characteristic of the E. coli E2plip domains) with threonine (as in the E. coli E2olip domain) did not affect the ability of the E2plip domain to undergo reductive acetylation by E1p (G39T domain; Figure 4). The G39T mutation did cause a small increase in the susceptibility of the E2plip domain to reductive succinylation by E1o, although this remained way below that of the wild-type E2o domain (Figure 5). The converse mutation in the E2o lipoyl domain (T42G) also resulted in a lower rate of reductive succinylation by its partner E1o (D.D.J., unpublished work). Thus, although this residue may help to ensure reductive acylation of the B. stearothermophilus E2p domain and possibly the E. coli E2o lipoyl domain by their respective E1 components, it does not do so for the E. coli E2p lipoyl domain.

The extent of reductive acetylation of the E. coli E2plip domain after prolonged exposure to E1p was also found to be affected by modification of the loop sequence. Exchanging the loop sequence for that of the E2olip domain, as with replacing the GDE sequence by SAG, led to a lower level of reductive acetylation than that obtained with the wild-type domain (Figure 4). This would suggest that the surface loop has an effect upon the stability of the thioester bond linking the acetyl group to the nearby dihydrolipoyl-lysine residue. The succinyl-dihydrolipoyl group in the E. coli (Collins \& Reed, 1977) and A. vinelandii (Berg et al., 1998) 2OGDH complexes has previously been reported to be somewhat unstable. In the H-protein of the pea leaf glycine cleavage system, the oxidized lipoyl group is immobilized by interactions with the protein (Pares et al., 1994), but changes position and retreats into a cleft on the protein surface after becoming reduced and aminomethylated as part of the overall reaction (Cohen-Addad et al., 1995). This appears to be required to protect the unstable catalytic intermediate, as formulated by the "hot potato" hypothesis of multienzyme complex mechanisms (Perham, 1975; Perham \& Reche, 1998). Although there is no evidence for a comparably complete immobilization of the lipoyl-lysine residue on the surface of a lipoyl domain in a 2-oxo acid dehydrogenase complex, it is conceivable that the surface loop plays an important part in stabilizing the thioester intermediate and that the lipoyllysine swinging arm is not as freely swinging as hitherto believed.

Deletion of four residues in the surface loop in the E. coli E2plip domain led to complete loss of 
the ability to become reductively acetylated (LD domain; Figure 4), as observed also with the B. stearothermophilus E2p lipoyl domain (Wallis et al., 1996). However, the E. coli E2plip LD domain does not appear to be fully folded. This perturbation in the structure could explain the loss of catalytic activity, as already proposed above for the diminished rate of lipoylation by LplA that accompanied it.

The present work has shown that the exposed surface loop between $\beta$-strands 1 and 2 is important in the interaction of the lipoyl domain with its partner E1. NMR spectroscopy has indicated that there may be direct contact between the loop and E1 in the B. stearothermophilus PDH complex (Wallis et al., 1996; Howard et al., 1999). For reductive acetylation or succinylation of a lipoyl domain by its partner E1 to take place, a precise conformation of the lipoyl domain and a fit to E1 may be required. Even a minor deviation from this may hinder the interaction (as indicated by the E2plip LS mutants), whereas a more significant deviation will lead to a total loss of recognition (as indicated by the E2plip LD mutant). Given that the E2plip LD domain has formed something of a lipoyllysine $\beta$-turn (see above), this structural feature alone is evidently not sufficient as a means of presenting the lipoyl-lysine residue to the active site of E1 for catalysis to occur. What is also clear is that the surface loop neighbouring the lipoyl-lysine $\beta$-turn in space is not the sole determinant in recognition of the lipoyl domain by E1 and that different lipoyl domains are recognised by their partner E1 in different ways.

\section{Materials and Methods}

\section{Materials}

Sodium $\left[2-{ }^{14} \mathrm{C}\right]$ pyruvate and sodium $\left[5-{ }^{14} \mathrm{C}\right] 2$-oxoglutarate were obtained from NEN Research Products and American Radiolabels Inc., respectively. All restriction enzymes were purchased from NE Biolabs and Pharmacia. Oligonucleotides were synthesized by Dr Charles Hill of the Protein and Nucleic Acid Chemistry Facility (PNAC), Department of Biochemistry, University of Cambridge. All other chemicals used were of analytical grade. E. coli lipoate protein ligase A (LplA) was kindly prepared by Mr C. Fuller as described elsewhere (Reche et al., 1998). E. coli E1p and E. coli E1o are both the products of genes over-expressed in E. coli E1o was a kind gift from Dr P.M. Ricaud.

\section{Protein engineering of the E2p domain}

Site-directed mutagenesis was carried out by splicing-overlap-extension (Ho et al., 1989; Horten et al., 1989) on a sub-gene encoding the innermost lipoyl domain of E. coli E2p (E.L. Roberts \& R.N.P., unpublished work), placed in the pET11c over-expression plasmid (Novagen) as for the E2o domain (Ricaud et al., 1996). Forward and reverse primers for PCR, based upon the pET11c DNA sequence flanking the insert, were used in conjunction with the mutagenesis primers. Sites for the restriction enzymes NcoI and
EcoNI were introduced into the E2p sub-gene by sitedirected mutagenesis, such that they flanked the DNA sequence encoding the surface loop between $\beta$-strands 1 and 2 of the domain. The PCR fragment containing the mutated sub-gene was digested with BamHI and inserted between the EcoRV and BamHI sites of pBluescript $\mathrm{SK}(+/-)$. The sub-gene was then digested with NcoI and EcoNI to remove the DNA encoding the loop region. Insertion of new loop sequences was carried out by cassette mutagenesis (Wells et al., 1985), annealing complementary oligonucleotides and ligating them into the sub-gene using T4 DNA ligase (Promega). For over-expression, the engineered sub-gene was removed from pBluescript SK by digestion with BamHI and NdeI and inserted back into pET11c cut with the same enzymes. All DNA was sequenced to confirm the identity of the intended products (kindly carried out by Mr J. Lester and Ms K. Pennock of the DNA Sequencing Facility, Department of Biochemistry, University of Cambridge). Wild-type and mutant lipoyl domains were over-expressed in E. coli BL21(DE3) cells and purified (as apo-forms) as described by Dardel et al. (1990) and Ricaud et al. (1996).

\section{Lipoylation of the E. coli lipoyl domains in vitro}

The apo-forms of the domains were lipoylated in vitro by exposure to E. coli LplA in the presence of lipoic acid, as follows. A reaction mixture containing $1.2 \mathrm{mM} \mathrm{ATP}$, $1.2 \mathrm{mM} \mathrm{MgCl} 2,0.5 \mathrm{mM}$ sodium lipoate (Sigma), $0.1 \mathrm{mg} /$ $\mathrm{ml} \mathrm{LplA}$ and 10-300 nmol of lipoyl domain in $20 \mathrm{mM}$ Tris- $\mathrm{HCl}(\mathrm{pH} 7.5)$, was incubated at $25^{\circ} \mathrm{C}$ for three hours (Lessard et al., 1998). The products were separated by anion exchange chromatography using a Resource- $\mathrm{Q}^{\mathrm{TM}}$ column (Pharmacia) developed with an ammonium bicarbonate gradient, essentially as described by Dardel et al. (1990). The lipoylated domains were bufferexchanged into $20 \mathrm{mM}$ sodium phosphate ( $\mathrm{pH} 7.0)$, and their identity, including the correct post-translational modification, was confirmed by means of electrospray mass spectrometry.

To assess the rate of lipoylation, the apo-form of the lipoyl domain $(50 \mu \mathrm{M})$ was incubated with $1.2 \mathrm{mM}$ ATP, $1.2 \mathrm{mM} \mathrm{MgCl}{ }_{2}$ and $0.6 \mathrm{mM}$ sodium lipoate in $20 \mathrm{mM}$ Tris- $\mathrm{HCl}$ ( $\mathrm{pH} \mathrm{7.5),} \mathrm{for} \mathrm{five} \mathrm{minutes} \mathrm{at} 25^{\circ} \mathrm{C}$. LplA was added to a concentration of $20 \mu \mathrm{g} / \mathrm{ml}$. At various times, samples $(0.4 \mathrm{nmol})$ of lipoyl domain were removed, mixed with a 20-fold molar excess of EDTA over $\mathrm{MgCl}_{2}$ and submitted to non-denaturing PAGE (20\% gels) to separate the apo- and holo-forms of the domain.

\section{Reductive acylation of the lipoyl domains}

Reductive acylation of the lipoyl domain in the presence of a given 2-oxo acid was measured using a modified version of the method described by Packman et al. (1984). A sample $(1 \mathrm{nmol})$ of lipoyl domain in $50 \mu \mathrm{l}$ of

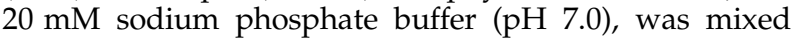
with $50 \mu \mathrm{l}$ of $20 \mathrm{mM}$ sodium phosphate buffer ( $\mathrm{pH} 7.0)$, containing $3 \mathrm{mM} \mathrm{NAD}{ }^{+}, 4 \mathrm{mM} \mathrm{MgCl}, 0.8 \mathrm{mM}$ ThDP and either $10 \mu \mathrm{g}$ of the relevant E. coli 2-oxo acid dehydrogenase complex or $3 \mu \mathrm{g}$ of E. coli E1p or E1o. After five minutes at $25^{\circ} \mathrm{C},{ }^{14} \mathrm{C}$-labelled 2-oxo acid was added to a concentration of $250 \mu \mathrm{M}$ and the mixture was left for a further 25 minutes at $25^{\circ} \mathrm{C}$. BSA $(10 \mu \mathrm{l}, 10 \mathrm{mg} / \mathrm{ml}$ in water) was added as a carrier and the proteins were then denatured by adding $1 \mathrm{ml}$ of ice-cold $10 \%(\mathrm{w} / \mathrm{v})$ 
trichloroacetic acid (TCA). After at least five minutes on ice, the precipitated protein was collected on $0.45 \mu \mathrm{m}$ filter discs (Millipore), and the tubes were washed twice with $10 \%$ TCA. The combined precipitate was washed with $20 \mathrm{ml}$ of ice-cold $10 \%$ TCA and dried before scintillation counting. All experiments were done in duplicate.

To estimate the rate of reductive acylation, $3 \mathrm{nmol}$ of lipoyl domain was incubated in a final volume of $300 \mu \mathrm{l}$

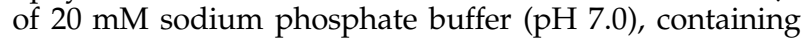
$0.2 \mathrm{mM}$ ThDP, $1 \mathrm{mM} \mathrm{MgCl}_{2}, 0.5 \mathrm{mM} \mathrm{NAD}{ }^{+}, \mathrm{BSA}$ $(0.3 \mathrm{mg} / \mathrm{ml})$ and $5 \mu \mathrm{g}$ of E. coli E1p or E1o. After five minutes at $25^{\circ} \mathrm{C},{ }^{14} \mathrm{C}$-labelled 2-oxo acid was added to a concentration of $200 \mu \mathrm{M}$. Samples $(45 \mu \mathrm{l})$ were withdrawn at various times and mixed with $1 \mathrm{ml}$ of $10 \%$ icecold TCA. The precipitates were collected and counted for radioactivity as described above. All experiments were done in duplicate.

\section{NMR spectroscopy}

All NMR spectra were obtained with a Bruker AM500 spectrometer at $298 \mathrm{~K}$. Homonuclear 1D spectra were recorded using 512 scans with 8096 data points in $t_{2}$. 2D NOESY spectra (Jeener et al., 1979; Kumar et al., 1980) were recorded using water suppression by presaturation and a mixing time of $100 \mathrm{~ms}$, and 32 scans with 2048 data points in the $t_{2}$ dimension and 512 increments in $t_{1}$. A spectral width of $8064.52 \mathrm{~Hz}$ was used in all experiments. Samples of lipoyl domain $(2-3 \mathrm{mM})$ were dissolved in $20 \mathrm{mM}$ sodium phosphate buffer ( $\mathrm{pH}$ 6.0), containing $10 \%(\mathrm{v} / \mathrm{v}){ }^{2} \mathrm{H}_{2} \mathrm{O}, 0.05 \%(\mathrm{w} / \mathrm{v})$ sodium azide and $40 \mu \mathrm{M}$ trimethylsilyl propionate (TSP) as the internal reference for ${ }^{1} \mathrm{H}$ chemical shift. Data were processed using the Azara suite of programs (W. Boucher, unpublished work).

\section{General techniques}

Protein concentrations were determined by amino acid analysis, kindly performed by $\mathrm{Mr}$ Peter Skerrit of the PNAC facility, University of Cambridge. Samples for mass spectrometry were prepared in aqueous acetonitrile $(50 \%, \mathrm{v} / \mathrm{v})$ containing formic acid $(1 \%, \mathrm{v} / \mathrm{v})$ and analysed on a VG BioQ quadrapole mass spectrometer using myoglobin as the standard. The $\mathrm{pH}$ values of the resolving and stacking gels for non-denaturing PAGE (Reche et al., 1998) were 7.5 and 5.5, respectively. The gels were stained with Coomassie brilliant blue. The extent of lipoylation was estimated by densitometric analysis of the bands representing the apo- and holoforms of the domain, the latter migrating more rapidly towards the anode (Reche et al., 1998).

\section{Acknowledgements}

We thank the Biotechnology and Biological Sciences Research Council for the award of a research grant (to R.N.P.) and the BBSRC and The Wellcome Trust for support of the core facilities of the Cambridge Centre for Molecular Recognition. D.D.J. is gratetul to the BBSRC and AdProTech for the award of a CASE Studentship. We thank Mr Paul Skelton and Mr John Hicks for advice on mass spectrometry, Dr Paul Ricaud for the gift of E1o and $\mathrm{Mr} \mathrm{C}$. Fuller for the preparation of LplA and skilled technical assistance.

\section{References}

Berg, A. \& de Kok, A. (1997). 2-Oxo acid dehydrogenase multienzyme complexes. The central role of the lipoyl domain. Biol. Chem. 378, 617-634.

Berg, A., Vervoort, J. \& de Kok, A. (1996). Solution structure of the lipoyl domain of the 2-oxoglutarate dehydrogenase complex from Azotobacter vinelandii. J. Mol. Biol. 261, 432-442.

Berg, A., Vervoort, J. \& de Kok, A. (1997). Three-dimensional structure in solution of the N-terminal lipoyl domain of the pyruvate dehydrogenase complex from Azotobacter vinelandii. Eur. J. Biochem. 244, 352360.

Berg, A., Westphal, A. H., Bosma, H. J. \& de Kok, A. (1998). Kinetics and specificity of reductive acylation of wild-type and mutated lipoyl domains of 2oxo acid dehydrogenase complexes from Azotobacter vinelandii. Eur. J. Biochem. 252, 45-50.

Cohen-Addad, C., Pares, S., Sieker, L., Neuburger, M. \& Douce, R. (1995). The lipoamide arm of the glycine decarboxylase complex is not freely swinging. Nature Struct. Biol. 2, 63-68.

Collins, J. H. \& Reed, L. J. (1977). Acyl group and electron pair relay system: a network of interacting lipoyl moieties in the pyruvate and alpha-ketoglutarate dehydrogenase complexes from Escherichia coli. Proc. Natl Acad. Sci. USA, 74, 4223-4227.

Dardel, F., Packman, L. C. \& Perham, R. N. (1990). Expression in Escherichia coli of a sub-gene encoding the lipoyl domain of the pyruvate dehydrogenase complex of Bacillus stearothermophilus. FEBS Letters, 264, 206-210.

Dardel, F., Davis, A. L., Laue, E. D. \& Perham, R. N. (1993). Three-dimensional structure of the lipoyl domain from Bacillus stearothermophilus pyruvate dehydrogenase multienzyme complex. J. Mol. Biol. 229, 1037-1048.

Graham, L. D. \& Perham, R. N. (1990). Interactions of lipoyl domains with the Elp subunits of the pyruvate dehydrogenase multienzyme complex from Escherichia coli. FEBS Letters, 262, 241-244.

Graham, L. D., Packman, L. C. \& Perham, R. N. (1989). Kinetics and specificity of reductive acylation of lipoyl domains from 2-oxo acid dehydrogenase complexes. Biochemistry, 28, 1574-1581.

Green, J. D. F., Perham, R. N., Ullrich, S. J. \& Appella, E. (1992). Conformational studies of the interdomain linker peptides in the dihydrolipoyl acetyltransferase component of the pyruvate dehydrogenase multienzyme complex of Escherichia coli. J. Biol. Chem. 267, 23484-23488.

Green, J. D. F., Laue, E. D., Perham, R. N., Ali, S. T. \& Guest, J. R. (1995a). Three dimensional structure of a lipoyl domain from the dihydrolipoyl acetyltransferase component of the pyruvate dehydrogenase multienzyme complex of Escherichia coli. J. Mol. Biol. 248, 328-343.

Green, D. E., Morris, T. W., Green, J., Cronan, J. E., Jr \& Guest, J. R. (1995b). Purification and properties of the lipoate protein ligase of Escherichia coli. Biochem. J. 309, 853-862.

Hirel, P. H., Schmitter, J. M., Dessen, P., Fayat, G. \& Blanquet, S. (1989). Extent of N-terminal methionine excision from Escherichia coli proteins is governed by the side-chain length of the penultimate amino acid. Proc. Natl Acad. Sci. USA, 86, 8247-8251.

Ho, S. N., Hunt, H. D., Horton, R. M., Pullen, J. K. \& Pease, L. R. (1989). Site-directed mutagenesis by 
overlap extension using the polymerase chain reaction. Gene, 77, 51-59.

Horton, R. M., Hunt, H. D., Ho, S. N., Pullen, J. K. \& Pease, L. R. (1989). Engineering hybrid genes without the use of restriction enzymes - gene-splicing by overlap extension. Gene, 77, 61-68.

Howard, M. J., Fuller, C., Broadhurst, R. W., Perham, R. N., Tang, J.-G., Quinn, J., Diamond, A. G. \& Yeaman, S. J. (1998). Three-dimensional structure of the major autoantigen in primary biliary cirrhosis. Gastroenterology, 115, 139-146.

Howard, M. J., Chauhan, H. J., Domingo, G., Fuller, C. \& Perham, R. N. (1999). Protein-protein interaction revealed by NMR $T_{2}$ relaxation experiments. The lipoyl domain and El component of the pyruvate dehydrogenase multienzyme complex of Bacillus stearotharmophilus. J. Mol. Biol. in the press.

Izard, T., Aevarsson, A., Allen, M. D., Westphal, A. H., Perham, R. N., de Kok, A. \& Hol, W. G. J. (1999). Principles of quasi-equivalence and Euclidean geometry govern the assembly of cubic and dodecahedral cores of pyruvate dehydrogenase complexes. Proc. Natl Acad. Sci. USA, 96, 1240-1245.

Jeener, J., Meier, J., Bachmann, P. \& Ernst, R. R. (1979). Investigation of the exchange processes by twodimensional NMR spectroscopy. J. Chem. Phys. 71, 323-341.

Kalia, Y. N., Brocklehurst, S. M., Hipps, D. S., Appella, E., Sakaguchi, K. \& Perham, R. N. (1993). The highresolution structure of the peripheral subunit-binding domain of dihydrolipoamide acetyltransferase from the pyruvate dehydrogenase multienzyme complex of Bacillus stearothermophilus. J. Mol. Biol. 230, 323-341.

Knapp, J. E., Mitchell, D. T., Yazdi, M. A., Ernst, S. R., Reed, L. J. \& Hackert, M. L. (1998). Crystal structure of the truncated cubic core component of the Escherichia coli 2-oxoglutarate dehydrogenase multienzyme complex. J. Mol. Biol. 280, 655-668.

Kraulis, P. J. (1991). MOLSCRIPT: a program to produce both detailed and schematic plots of protein structure. J. Appl. Crystallog. 24, 946-950.

Kumar, A., Ernst, R. R. \& Wuthrich, K. (1980). A twodimensional nuclear Overhauser enhancement experiment for the elucidation of complete protonproton cross-relaxation networks in biological macromolecules. Biochem. Biophys. Res. Commun. 95, 1-6.

Lessard, I. A. D., Domingo, G. J., Borges, A. \& Perham, R. N. (1998). Expression of genes encoding the E2 and E3 components of the Bacillus stearothermophilus pyruvate dehydrogenase complex and the stoichiometry of subunit interaction in assembly in vitro. Eur. J. Biochem. 258, 491-501.

Macherel, D., Bourguignon, J., Forest, E., Faure, M., Cohen-Addad, C. \& Douce, R. (1996). Expression, lipoylation and structure determination of recombinant pea H-protein in Escherichia coli. Eur. J. Biochem. 122, 1523-1530.

Mande, S. S., Sarfaty, S., Allen, M. D., Perham, R. N. \& Hol, W. G. J. (1996). Protein-protein interactions in the pyruvate dehydrogenase multienzyme complex: dihydrolipoamide dehydrogenase complexed with the binding domain of dihydrolipoamide acetyltransferase. Structure, 4, 277-286.

Mattevi, A., de Kok, A. \& Perham, R. N. (1992a). The pyruvate dehydrogenase multienzyme complex. Curr. Opin. Struct. Biol. 2, 877-887.
Mattevi, A., Obmolova, G., Schulze, E., Kalk, K. H., Westphal, A. H., de Kok, A. \& Hol, W. G. J. (1992b). Atomic structure of the cubic core of the pyruvate dehydrogenase multienzyme complex. Science, 255, 1544-1550.

Morris, T. W., Reed, K. E. \& Cronan, J. E. (1995). Lipoic acid metabolism in Escherichia coli - the LplA and LipB genes define redundant pathways for ligation of lipoyl groups to apoprotein. J. Bacteriol. 177, 1-10.

Packman, L. C., Perham, R. N. \& Roberts, G. C. K. (1984). Domain structure and ${ }^{1} \mathrm{H}-\mathrm{NMR}$ spectroscopy of the pyruvate dehydrogenase complex of Bacillus stearothermophilus. Biochem. J. 217, 219-227.

Pares, S., Cohen, Addad C., Sieker, L., Neuburger, M. \& Douce, R. (1994). X-ray structure determination at 2. 6-Angstrom resolution of a lipoate-containing protein - the H-protein of the glycine decarboxylase complex from pea leaves. Proc. Natl Acad. Sci. USA, 91, 4850-4853.

Perham, R. N. (1975). Self-assembly of biological macromolecules. Phil. Trans. Roy. Soc. London ser. B, 272, 123-136.

Perham, R. N. (1991). Domains, motifs, and linkers in 2oxo acid dehydrogenase multienzyme complexes: a paradigm in the design of a multifunctional enzyme. Biochemistry, 30, 8501-8512.

Perham, R. N. \& Reche, P. A. (1998). Swinging arms in multifunctional enzymes and the specificity of posttranslational modification. Biochem. Soc. Trans. 26, 299-303.

Quinn, J., Diamond, A. G., Masters, A. K., Brookfield, D. E., Wallis, N. G. \& Yeaman, S. J. (1993). Expression and lipoylation in Escherichia coli of the inner lipoyl domain of the E2 component of the human pyruvate dehydrogenase complex. Biochem. J. 289, 81-85.

Reche, P. \& Perham, R. N. (1999). Structure and selectivity in post-translational modification: attaching the biotinyl-lysine and lipoyl-lysine swinging arms in multifunctional enzymes. EMBO J. 18, 2673-2682.

Reche, P. A., Li, Y. L., Fuller, C., Eichhorn, K. \& Perham, R. N. (1998). Selectivity of post-translational modification in biotinylated proteins: the carboxy carrier protein of the acetyl-CoA carboxylase of Escherichia coli. Biochem. J. 329, 589-596.

Reed, L. J. \& Hackert, M. L. (1990). Structure-function relationships in dihydrolipoamide acyltransferase. J. Biol. Chem. 265, 8971-8974.

Reed, L. J., Leach, F. R. \& Koike, M. (1958). Studies on a lipoic acid activating system. J. Biol. Chem. 232, 123142.

Ricaud, P. M., Howard, M. J., Roberts, E. L., Broadhurst, R. W. \& Perham, R. N. (1996). Three-dimensional structure of the lipoyl domain from the dihydrolipoyl succinyltransferase component of the 2-oxoglutarate dehydrogenase multienzyme complex of Escherichia coli. J. Mol. Biol. 264, 179-190.

Robien, M. A., Clore, G. M., Omichinski, J. G., Perham, R. N., Appella, E., Sakaguchi, K. \& Gronenborn, A. M. (1992). Three-dimensional solution structure of the E3-binding domain of the dihydrolipoamide succinyltransferase core from the 2-oxoglutarate dehydrogenase multienzyme complex of Escherichia coli. Biochemistry, 31, 3463-3471.

Turner, S. L., Russell, G. C., Williamson, M. P. \& Guest, J. R. (1993). Restructuring an interdomain linker in the dihydrolipoamide acetyltransferase component of the pyruvate dehydrogenase complex of Escherichia coli. Protein Eng. 6, 101-108. 
Wallis, N. G. \& Perham, R. N. (1994). Structural dependence of post-translational modification and reductive acetylation of the lipoyl domain of the pyruvate dehydrogenase complex. J. Mol. Biol. 236, 209-216.

Wallis, N. G., Allen, M. D., Broadhurst, R. W., Lessard, I. A. D. \& Perham, R. N. (1996). Recognition of a surface loop of the lipoyl domain underlies substrate channelling in the pyruvate dehydrogenase multienzyme complex. J. Mol. Biol. 263, 436-474.

Wells, J. A., Vasser, M. \& Powers, D. B. (1985). Cassette mutagenesis - an efficient method for the generation of multiple mutations at defined sites. Gene, 34, 315323.

Edited by A. R. Fersht

(Received 22 July 1999; received in revised form 14 October 1999; accepted 29 October 1999) 